\title{
Modeling analysis of ultrasonic attenuation and angular scattering measurements of suspended particles
}

\author{
Peter Fleckenstein and Giuseppe Stortia) \\ Institute for Chemical and Bioengineering, ETH Zürich, Vladimir Prelog Weg 1, 8093 Zürich, Switzerland \\ Fabian Deschwanden and Peter Gruber \\ Institute of Mechanical Engineering, Hochschule Luzern, Technikumstrasse 21, 6048 Horw, Switzerland \\ Marco Lattuada \\ Department of Chemistry, University of Fribourg, Chemin du Musée 9, 1700 Fribourg, Switzerland
}

(Received 27 June 2017; revised 24 January 2018; accepted 28 January 2018; published online 20 February 2018)

A combination of two models previously developed by Faran, and Atkinson and Kytömaa (FaranAK model) was used to calculate the ultrasonic attenuation and the backscattering signal of a suspension of particles. The model of Atkinson and Kytömaa yielded the viscoelastic contributions while the model of Faran yielded the scattering contribution. A comparison with the more fundamental model by Epstein, Carhart, Allegra, and Hawley validated the combination, where the combination used here proved to be computationally less intensive and more stable. The Faran-AK model outputs were also compared with ultrasound measurements of glass beads with two different particle size distributions and varying concentrations. The comparison showed a very reasonable agreement of model and experiment. (C) 2018 Acoustical Society of America.

https://doi.org/10.1121/1.5024233

[JFL]

Pages: 1049-1063

\section{INTRODUCTION}

Acoustic spectroscopy is a powerful technique, commonly used to extract several physical properties of colloidal dispersions. ${ }^{1}$ In particular, particle size distribution and zeta potential $^{2}$ can be determined. The method is also conventionally used to control and monitor on-line certain processes, such as crystallization. ${ }^{3}$ While other methods, usually based on light scattering, can also be used to obtain analogous information, they suffer from the disadvantage of requiring high dilutions of the samples. ${ }^{4}$ Conversely, acoustic spectroscopy offers the enormous advantage of allowing the investigation of highly concentrated suspensions, thus providing more realistic data for all applications where dilution might significantly alter the conditions of the suspension. ${ }^{1,5}$ Furthermore, acoustic measurements can also be performed under certain conditions through reactors or pipes walls, thus greatly enhancing the applicability of this technique in industrial settings. ${ }^{6}$

Conventional acoustic spectroscopy operates in forward scattering mode. This means that it measures the attenuation of ultrasound waves that propagate through a sample as a function of the wave frequency, and also measures the sound velocity through the suspension. By applying wellestablished theories, ${ }^{7-14}$ particle size and size distributions can be obtained from the analysis of attenuation and the sound velocity spectra.

Direct acoustic backscattering is one of the most investigated alternative geometries. Measuring in backscattering mode can be advantageous in setups where a forward measurement is either impractical or completely impossible. One

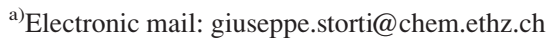

of the most established uses of ultrasound backscattering is the measurement of flow velocity and turbulence by tracking suspended particles, a method known as acoustical Doppler profiling (ADP). ${ }^{15-18}$

There is a multitude of examples for the use of direct acoustical backscattering in marine geology or other civil engineering areas to measure suspended sediment concentration (SSC). ${ }^{19-33}$ Backscattering measurements have the great advantage of having a very large penetration depth (several meters). ${ }^{34}$ Therefore, ultrasound backscattering measurements are becoming an important tool for sediment dynamics studies and monitoring of sediment transport. ${ }^{35-39}$ Costa et al. used this type of measurement for sizing silt. ${ }^{40}$ Compared to the measurement of SSCs from direct attenuation, ${ }^{5}$ which requires a broad spectrum of frequencies, only one frequency was used by Costa et al. The drawback of this measurement is that the penetration depth of the measurement is dependent on factors like concentration, frequency, and particle size of the suspension and can therefore be very short.

The use of bistatic or angular backscattering in acoustic spectroscopy has also been investigated, even though less thoroughly than in the case of forward scattering configurations. Experiments for the fundamental understanding of angular sound scattering from spheres have been performed in the past ${ }^{41-44}$ and even irregular particles were considered. ${ }^{45-47}$ Most work on the bistatic case is based on the extensive work by Hay. This includes the assessment of the sound scattering and absorption theory, ${ }^{13}$ experimental studies, ${ }^{48-52}$ and rigorous comparison of data with the literature model. ${ }^{53}$ Approaches for the inversion of backscattering data $^{54-60}$ were also discussed for these systems. Recently, 
such setup has been proposed by Moore et al. as an alternative to the direct backscattering mode. ${ }^{61}$ In this manner, the intensity of the scattered ultrasonic signal was recorded at a few scattering angles, in the range from $95^{\circ}$ to $165^{\circ}$, and also at a few frequencies, from 1.5 to $4 \mathrm{Mhz}$. The authors measured the angular backscattering of solid particles from a turbulent jet of particles at different frequencies. They used the data to extract the particle size, and obtained a good qualitative agreement with the experimental data.

The goal of this work is to show how backscattering data obtained at only one scattering angle and a few frequency values can be well interpreted using a combination of literature models. For this purpose, the angular ultrasound scattering signals of well characterized glass bead samples with different weight fractions and two different average sizes have been recorded at three different frequencies. The data, combined with forward scattering attenuation signals, have been treated using a combination of Faran's rigorous scattering model ${ }^{14}$ with the model developed by Atkinson and Kytömaa. ${ }^{9,10}$ The modeling approach developed was also tested with more rigorous models, to justify its use. It has been shown that the proposed approach can quantitatively interpret the experimental data.

\section{THEORY}

In order to compare model predictions with experimental data, a general expression for the pressure of the acoustic wave scattered from a particle and reaching the detector is needed. The model used in this work was already reported by Moore and Hay. ${ }^{61}$ Its derivation can be found in Appendix B. The model provides the square average pressure of an ultrasound wave scattered by a suspension of particles, impinging on a detector located at an angle $\theta$ with respect to the direction of the ultrasound emitting source,

$$
\begin{aligned}
\left|p_{s}\right|^{2}(t)= & \frac{3 M p^{* 2} r^{* 2}}{4 \pi\left\langle a^{3}\right\rangle \rho_{s}} \int_{-\pi}^{\pi} \int_{-\pi / 2}^{\pi / 2} \int_{R(t)}^{R(t)+\Delta R}\left[\frac{\left\langle f_{\infty}(\theta, a) a\right\rangle^{2}}{\left(r_{i} r_{s}\right)^{2}}\right. \\
& \left.\times\left(D_{i} D_{s}\right)^{2} \exp \left(-2\langle\alpha\rangle\left(r_{i}+r_{s}\right)\right) r_{i}^{2} \frac{\partial r_{i}}{\partial R} \cos \varphi_{i}\right] \\
& \times d r_{i} d \varphi_{i} d \theta_{i}
\end{aligned}
$$

where $M$ is the particles mass concentration, $p^{*}$ is a reference pressure at a reference distance $r^{*}, a$ is the particle radius, $\left\langle a^{3}\right\rangle$ is the average volume of the SSC of particles, $\rho_{s}$ is the particle density, $r_{i}$ and $r_{s}$ are the distances from transmitter to particle and from particle to receiver, respectively, $D_{i}$ and $D_{s}$ are the transducer functions of transmitter and receiver, $R$ is the sum of $r_{i}$ and $r_{s}$ and $\Delta R$ is the length of the acoustic pulse multiplied by the sound speed in water, $f_{\infty}$ is the far field function, and $\alpha$ is the attenuation. $f_{\infty}$ describes the scattering amplitude of a plane wave from a spherical particle as a function of the scattering angle. In this work, Faran's model has been used to calculate the far field function. The attenuation, representing the ability of particles to reduce the amplitude of a sound wave, has been calculated using a combination of Faran's model, valid for high sound frequency values, and the Atkinson and Kytömaa (AK) model, taking into account viscous effects. A justification of these choices is given in the Sec. IV.

Special note has to be taken for the expressions of $\langle\alpha\rangle$ and $\left\langle\left|f_{\infty}(\theta, a) a\right|^{2}\right\rangle$. The first is the average attenuation over the particle size distribution $n(a)$ (which is the number of particles having a size comprised between $a$ and $a+d a$ ), given by

$$
\langle\alpha\rangle=\frac{\int_{a} n(a) \alpha d a}{\int_{a} n(a) d a} .
$$

The second is the average of the far field function over the entire particle size distribution (PSD),

$$
\left\langle\left|f_{\infty}(\theta) a\right|^{2}\right\rangle=\int_{a} n(a) a^{2}\left|f_{\infty}\left(k_{c} a, \theta\right)\right|^{2} d a .
$$

Moore and Hay were using the simplification

$$
\left\langle\left|f_{\infty}(\theta, a) a\right|^{2}\right\rangle=\left\langle\left|f_{\infty}\left(\theta_{0}, a\right) a\right|^{2}\right\rangle,
$$

which is only valid for narrow beam widths. This approximation was possible because the solid particles measured in that work were concentrated in a jet around the crossing of the transducers main axis, with an angle between their axes equal to $\theta_{0}$. In this work, the complete model without simplifications was used, since a homogenous suspension of particles with a constant SSC was analyzed. The attenuation has been computed as the sum of the viscoelastic contributions given by the model of Atkinson and Kytömaa and the scattering contribution given by the Faran model

$$
\alpha_{\text {ex }}=\alpha_{\text {Kytomaa }}+\alpha_{\text {Faran }} \text {. }
$$

The derivation of Eq. (1) is found in Appendix A. The model output is the squared pressure amplitude, which can be correlated to the experimentally recorded voltage amplitude by a linear constant.

Attenuation measurements were performed simultaneously with backscattering measurements, and the excess attenuation was recovered. The same approach as Costa et $a l .^{40}$ was used to compute the excess attenuation,

$$
\alpha_{e x}=-\frac{1}{L_{p a t h}} \ln \left(\frac{V_{\exp }}{V_{\exp , 0}}\right)
$$

where $V_{\text {exp }}$ is the measured voltage of the ultrasonic receiver, $V_{\text {exp }, 0}$ is the measured voltage of the receiver without any suspended sediment, and $L_{\text {path }}$ was calculated by measuring the time the signal was traveling from one sensor to the other and multiplying that by the speed of sound in pure water.

\section{EXPERIMENTAL}

\section{A. Materials}

Glass beads were supplied by Abritec AG and analyzed by small angle static light scattering using a Malvern Mastersizer 2000. Particles with an average diameter of 

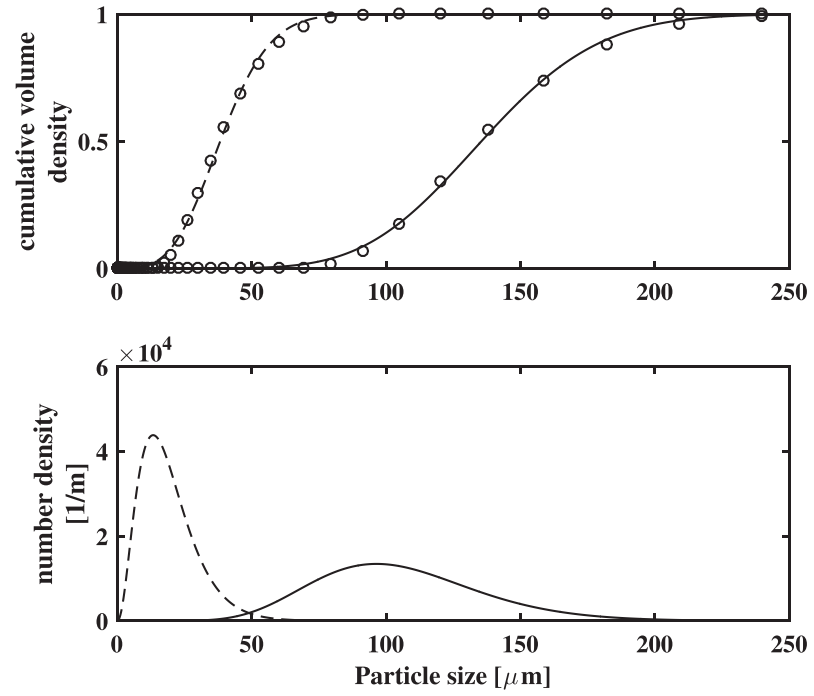

FIG. 1. Particle size distribution of the two different glass beads used for the experiments. Average diameters: $20 \mu \mathrm{m}$ (-) and $105 \mu \mathrm{m}$ (-). Cumulative volume density distributions were extracted from small angle light scattering data $(\bigcirc)$.

$20 \pm 10 \mu \mathrm{m}$ and $105 \pm 30 \mu \mathrm{m}$ were used. Their size distributions are shown in Fig. 1.

\section{B. Equipment}

The setup used for the backscattering measurements was a Plexiglas tank with diameter of $0.5 \mathrm{~m}$ and height of $1.2 \mathrm{~m}$. The ultrasound transducers were attached to the metal rig sketched in Fig. 2. Distances and angles of all the transmitters and receivers are reported in Table I. The sensor paths are indicated in the figure by black dotted lines for the attenuation pathway (channel 1 to channel 2) and the angular scattering (channel 1 to channel 3). Ultrasonic transducers with a frequency of $0.5,2$, and $4 \mathrm{MHz}$ were supplied by Basler Medizintechnik AG. Transducers with a frequency of $1 \mathrm{MHz}$ were supplied by Rittmeyer AG. A RiMo-emitterreceiver module also from Rittmeyer was used for emitting and receiving signals from the transducers. The sampling
TABLE I. Distances and angles between transmitter (channel 1) and the receivers (channels 2 and 3 ).

\begin{tabular}{lccccc}
\hline \hline \multirow{2}{*}{$\begin{array}{l}\text { Frequency } \\
{[\mathrm{MHz}]}\end{array}$} & \multicolumn{2}{c}{ Channel 1-channel 2 } & & \multicolumn{2}{c}{ Channel 1-Channel 3 } \\
\cline { 2 - 2 } \cline { 5 - 6 } \cline { 5 - 6 } & Distance [m] & Angle & & Distance [m] & Angle \\
\hline 0.5 & Not available & & & 0.228 & $90^{\circ}$ \\
1 & Not available & & & 0.396 & $90^{\circ}$ \\
2 & 0.224 & $0^{\circ}$ & & 0.317 & $90^{\circ}$ \\
4 & 0.224 & $0^{\circ}$ & & 0.317 & $90^{\circ}$ \\
\hline \hline
\end{tabular}

frequency was $50 \mathrm{MHz}$. Particles were suspended using a Grundfos pump (maximal flowrate $3 \mathrm{~L} / \mathrm{s}$ ).

\section{Backscattering and attenuation measurements}

The tank was filled with tap water and left at rest until full degassing. A schematic of the measurement setup with all the sensors is depicted in Fig. 2. The pump was switched on and set to its maximal power. A signal was emitted from transducer one $(\mathrm{CH} 1)$ and simultaneously recorded by channels 2 and 3 (CH2 and $\mathrm{CH} 3)$. In this configuration, 100 signals per frequency were recorded.

Then, a defined amount of glass beads with an average diameter of $20 \mu \mathrm{m}$ was added to the tank in order to increase the SSC. Again 100 signals were recorded and a small quantity of suspension (roughly $100 \mathrm{~mL}$ ) was sampled by a syringe at the height of the transmitters to be used for gravimetric measurement of the solid content (below indicated as SSC).

Solid addition and subsequent measurement were repeated until a nominal concentration of $10 \mathrm{~g} / \mathrm{L}$ was reached. The tank was emptied, cleaned of all glass beads and the same type of experiment was repeated with particles with larger average diameter $(105 \mu \mathrm{m})$.

\section{Data treatment}

Every recorded measurement signal was treated according to the following procedure. A preliminary noise removal was performed by treating the data with a Butterworth
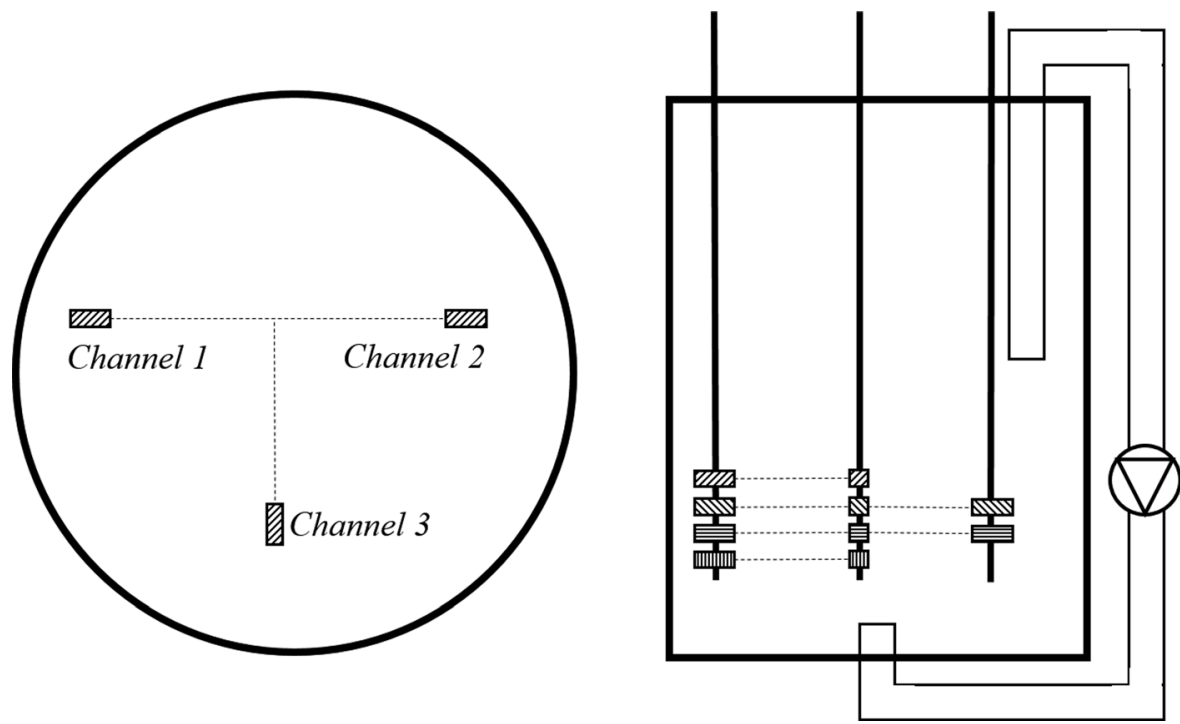

FIG. 2. Schematic of the top view (left) and side view (right) tank setup. The four sensors are arranged as follows: $\mathbf{Z Z} 1 \mathrm{MHz}, \mathbf{D V} 2 \mathrm{MHz}$, $4 \mathrm{MHz}$, IIIII $0.5 \mathrm{MHz}$. Channel 1 is the transmitter, while channels 2 and 3 are receiving the direct and the angular scattered signal, respectively. Angles and distances between the channels are reported in Table $\mathrm{I}$. 
TABLE II. Cutoff frequencies used in the Butterworth filter.

\begin{tabular}{lcc}
\hline \hline $\begin{array}{l}\text { Transducer } \\
\text { frequency }[\mathrm{MHz}]\end{array}$ & $\begin{array}{c}\text { Lower cutoff } \\
\text { frequency [MHz] }\end{array}$ & $\begin{array}{c}\text { Higher cutoff } \\
\text { frequency [MHz] }\end{array}$ \\
\hline 0.5 & 0.25 & 1 \\
1 & 0.5 & 2 \\
2 & 1 & 4 \\
4 & 2 & 8 \\
\hline \hline
\end{tabular}

bandpass filter. The frequency bandwidths used for the different frequencies are reported in Table II. The square of the signal was taken and in order to remove noise, the baseline was adjusted to have an average value of $0 \mathrm{~V}^{2}$. The signal was then integrated over time, where the integration limits were dependent on the frequency and the angle. The integration limits are reported in Tables III and IV. An example of the signal treatment can be seen in Fig. 3. Finally, an average of 100 signals was used for the comparison with the model.

\section{E. Simulation of the backscattering pressure amplitude}

Backscattering signals were calculated using MATLAB ${ }^{\circledR}$ (The Mathworks). The physical parameters for the continuous phase (density, sound speed, attenuation) and dispersed phase (density, sound speed, Poisson ratio) were taken from the literature. ${ }^{14}$ It was assumed that glass beads have the same physical properties as amorphous silica. The parameters defining the particle size distribution of the glass beads were obtained from small angle light scattering. The input lengths and angles were measured at the setup. As input concentration, the values determined by gravimetric analysis were used. As input frequencies, the nominal frequencies of the transducer were taken. As modeling pulse length, the real length of the acoustic pulse was used. The transducer function was approximated as a Gaussian distribution function, the standard deviation of which was determined so that the area under the curve was the same as for the real transducer function (Appendix B). The radius of the membrane needed for the transducer function was measured for each transducer. Using Eq. (1), the square pressure of the signal was then integrated using the MATLAB function INTEGRAL3. The signal itself was then further integrated over time. The square root of the integrated signal was then multiplied by the calibration constant.

\section{RESULTS AND DISCUSSION}

\section{A. Assessment of the theory used}

Several theories were developed in the past to describe the acoustical behavior of particulate suspensions, with

TABLE III. Integration limits for backscattering signals recorded at $90^{\circ}$.

\begin{tabular}{lcc}
\hline \hline $\begin{array}{l}\text { Transducer } \\
\text { frequency }[\mathrm{MHz}]\end{array}$ & $\begin{array}{c}\text { Lower integration } \\
\text { bound }[\mu \mathrm{s}]\end{array}$ & $\begin{array}{c}\text { Upper integration } \\
\text { bound }[\mu \mathrm{s}]\end{array}$ \\
\hline 0.5 & 185 & 240 \\
1 & 165 & 200 \\
2 & 120 & 150 \\
4 & 110 & 170 \\
\hline \hline
\end{tabular}

TABLE IV. Integration limits for backscattering signals recorded in forward scattering mode.

\begin{tabular}{lcc}
\hline \hline $\begin{array}{l}\text { Transducer } \\
\text { frequency }[\mathrm{MHz}]\end{array}$ & $\begin{array}{c}\text { Lower integration } \\
\text { bound }[\mu \mathrm{s}]\end{array}$ & $\begin{array}{c}\text { Upper integration } \\
\text { bound }[\mu \mathrm{s}]\end{array}$ \\
\hline 0.5 & 140 & 230 \\
1 & 195 & 230 \\
2 & 140 & 155 \\
4 & 140 & 148 \\
\hline \hline
\end{tabular}

particular focus on spherical particles. The approaches can be divided into fundamental models, describing in detail the scattering of ultrasound waves by particles, and coupledphase models, which describe the ultrasound scattering of suspensions treated as continuous systems. On the fundamental side, the most rigorous model is the one developed by Epstein, Carhart, Allegra, and Hawley (ECAH). ${ }^{7,8}$ It describes the behavior of a spherical particle (or droplet) in a suspension exposed to a sound wave, and was developed accounting for all the effects a plane acoustic wave has on the particle. These effects include thermal, viscous and scattering effects. A simplified version, neglecting thermal effects was developed by Hay and Mercer (HM). ${ }^{13}$ A further simplification neglects the viscous interactions of the particle with the solvent, leading to the oldest rigorous model describing only the scattering of sound from a solid sphere. This model, which was developed by Faran in the 1950s (Ref. 14) with some small corrections implemented by Hickling, ${ }^{62}$ is easy to implement, computationally very efficient, and numerically stable. An additional advantage is that it only requires the knowledge of the elastic properties
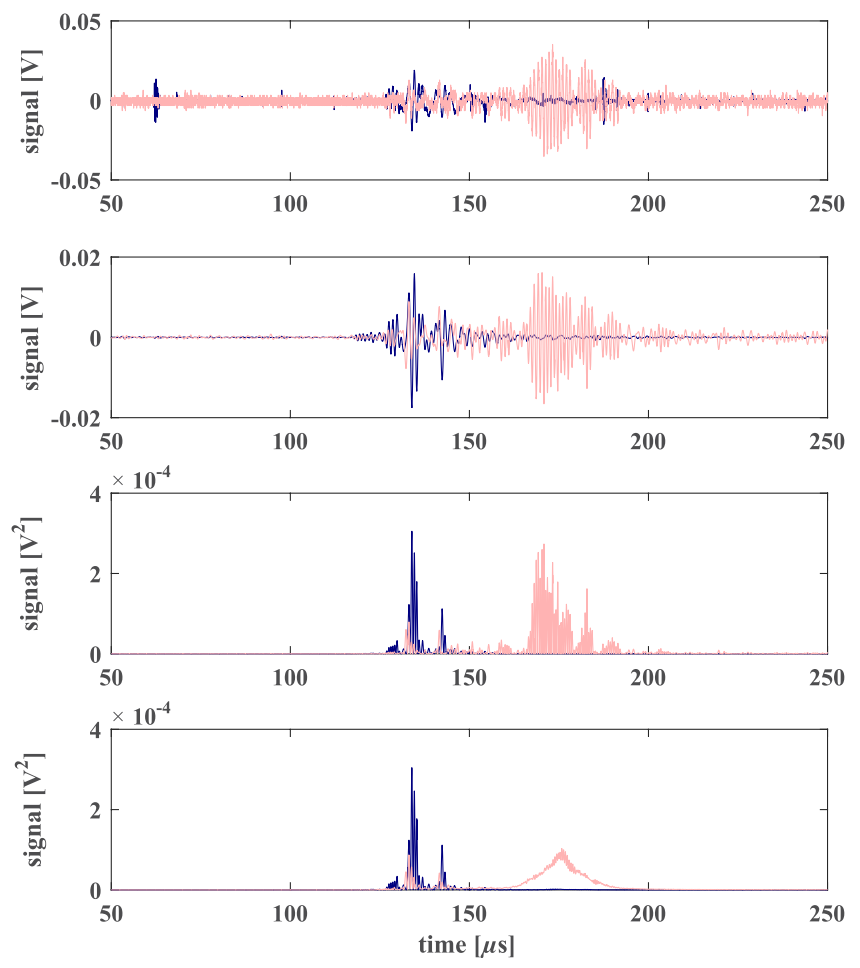

FIG. 3. (Color online) Signal treatment example for backscattering signal at $1 \mathrm{MHz}$. Dark blue $0 \mathrm{~g} / \mathrm{L} \mathrm{SSC}$. Light red $8 \mathrm{~g} / \mathrm{L} \mathrm{SSC}$. From top to bottom: raw signal obtained from the oscilloscope, Signal filtered with a Butterworth filter, squared signal, average of 100 squared signals. 


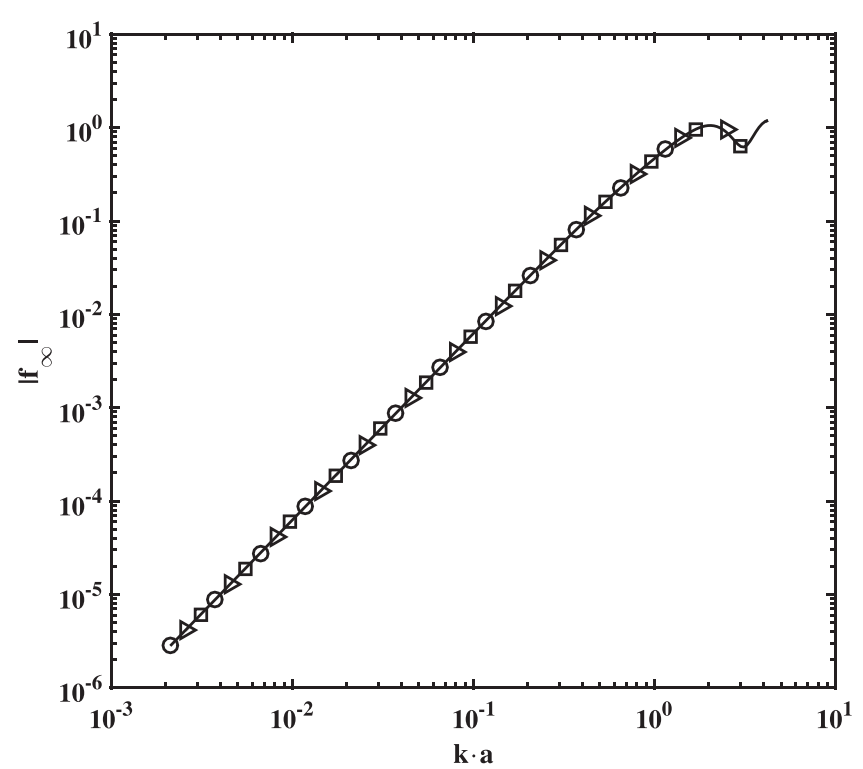

FIG. 4. Comparison of the far field functions for three models: Faran $(\triangle)$, HM ( $\square)$, ECAH (O). The solid line corresponds to the Faran model.

of the particle and the sound velocity of the solvent in which they are dispersed. On the other hand, it is only valid in the short wavelength regime (SWR), where scattering effects dominate the acoustic behavior.

A first task of this work was the comparison of the prediction of the three models, in order to assess the relative importance of the different effects, and choose the most suitable model. The values for the $90^{\circ}$ angular scattering far field function amplitude are depicted in Fig. 4, as a function of the particle size, made dimensionless with the wave vector modulus. The calculations made by using the ECAH model, HM model, and Faran model, overlap for all three cases. This indicates that the far field function is dominated by the scattering contribution, which is considered by all three models. It therefore makes sense to use the simplest of

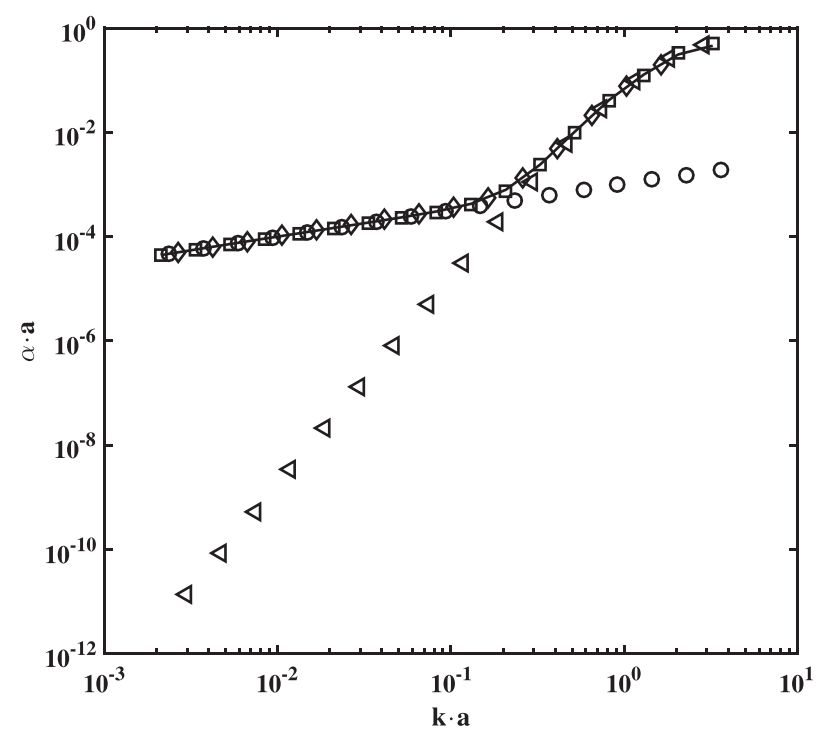

FIG. 5. Comparison of attenuation for four models: Faran $(\triangle), H M(\square)$, $\mathrm{ECAH}(\diamond)$, AK $(\bigcirc)$. The solid line depicts the combination of Faran and AK model.

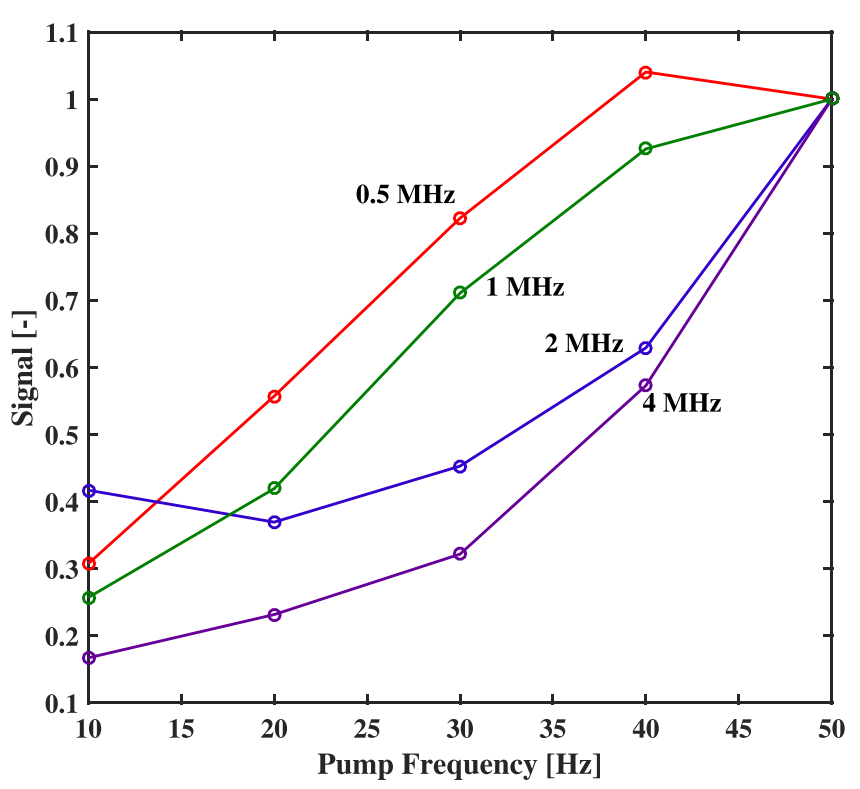

FIG. 6. (Color online) Normalized signal for different frequencies at $0 \mathrm{~g} / \mathrm{L}$ SSC in non-degassed water. It is evident, that the signal intensity of the backscattered signal increases with the pump frequency. This is not the case for the degassed water.

the three, i.e., the Faran model, to compute the far-field function.

The attenuation, which describes the reduction of signal intensity over length, is shown in Fig. 5. Here the Faran model is deviating from HM and ECAH models in the long wavelength regime (LWR), in which viscous effects dominate, while the predictions of the HM and ECAH models overlap, indicating that thermal effects are completely negligible for the system investigated in this work. Instead of using the HM model, which is numerically quite cumbersome, we decided to combine Faran model with the model by Atkinson and Kytomaa, which is a coupled-phase model, which is designed to explicitly capture the viscous dissipation effects in the attenuation.

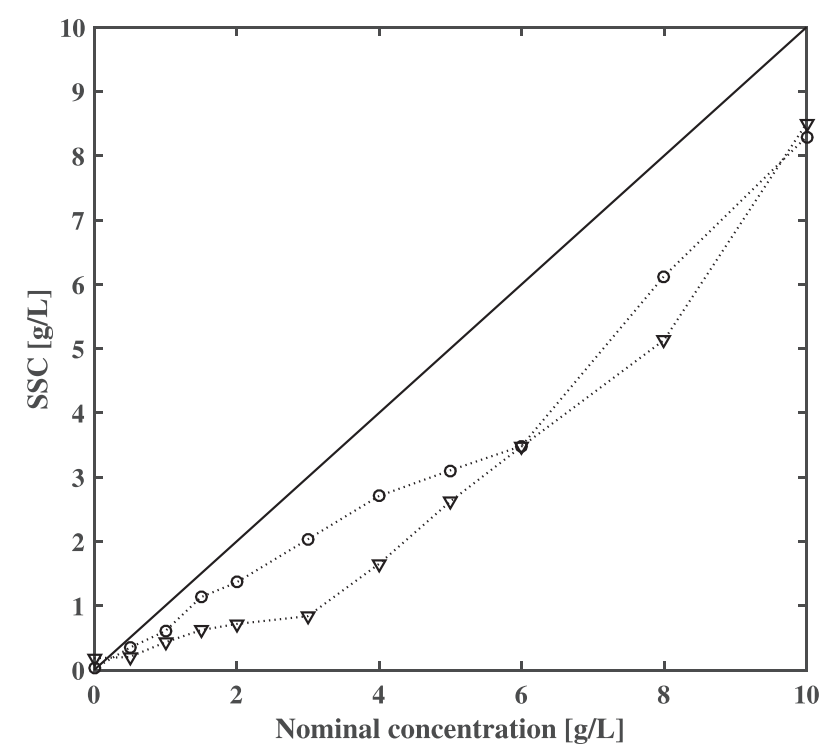

FIG. 7. Gravimetric measurement of SSC for the scattering measurements for particles of size $20 \mu \mathrm{m}(\bigcirc)$ and $105 \mu \mathrm{m}(\nabla)$. 

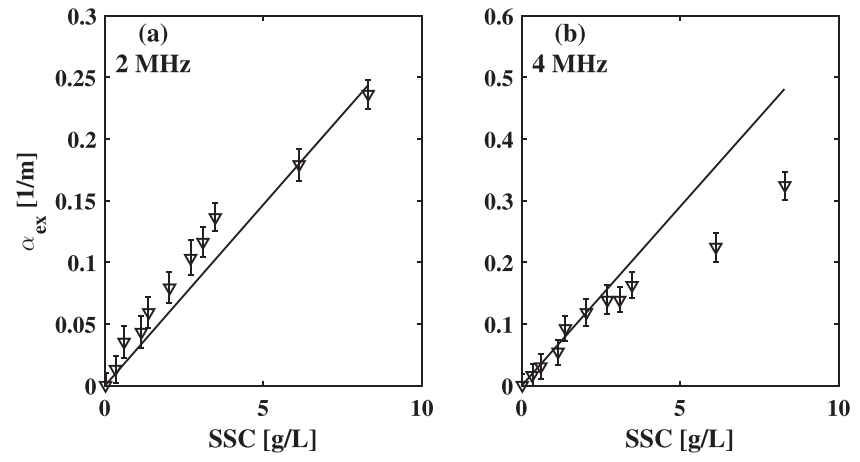

FIG. 8. Experimental ( $\nabla$ ) and predicted (-) excess attenuation of $20 \mu \mathrm{m}$ particles at different SSC values for $2 \mathrm{MHz}$ (a) and $4 \mathrm{MHz}$ (b). Model predictions were done with the combined Faran-AK model.

Among the various alternatives available in the literature to describe the attenuation for the viscous regime, ${ }^{63,64}$ we have chosen the model of Atkinson and Kytömaa., ${ }^{9,10,40}$ Figure 6 shows that the predictions of Kytömaa's model overlap very well with those of HM and ECAH models in the LWR, while deviations are observed in the high wavelengths regime, where scattering becomes the dominant mechanism. In fact, adding the attenuation computed by means of Faran model to the one from Atkinson and Kytömaa (AK), one obtains almost identical results as those predicted by the HM and ECAH models, with the added benefit of numerically stable and much faster calculations of the needed quantities. Therefore, in this work we only compare the experimental data to the combined Faran-AK model. The corresponding relevant equations of both models can be found in Appendix A.
TABLE V. Calibration constants for each frequency.

\begin{tabular}{lcccc}
\hline \hline Sensor frequency [MHz] & 0.5 & 1 & 2 & 4 \\
\hline Calibration constant $20 \mu \mathrm{m}$ particles [V/Pa] & 80000 & 10000 & 5500 & 1500 \\
Calibration constant $105 \mu \mathrm{m}$ particles [V/Pa] & 55000 & 3500 & 3000 & 650
\end{tabular}

\section{B. Discussion of the experimental results}

Several important features of the experimental procedures will be discussed. First and foremost, degassing the water prior to the experiment was found to be crucial to obtain reliable data. Fresh tap water contains a significant amount of dissolved gas, which leads to the formation of microbubbles under the shear generated by the pump. The influence of air bubbles has been discussed by Povey ${ }^{65}$ and others. ${ }^{66}$ It is known that the simultaneous scattering from air bubbles renders impossible the measurement of the scattering signal from dispersed particles (Fig. 6).

Degassing the water for a week improved substantially the quality of both backward and forward signals. Second, it was found that the large density contrast between sand and water led to difficulties in the complete suspension of all added particles. Therefore, it was necessary to use gravimetric measurements to determine the actual SSC, which was found to be well below the nominal one, as shown in Fig. 7. Therefore, the actual concentration was used as a model input for both forward and backward scattering calculations.

The excess attenuation and the backscattered signal could be modeled using the experimentally obtained distributions of the glass beads. The experimental excess attenuation and the corresponding model predictions for the case of
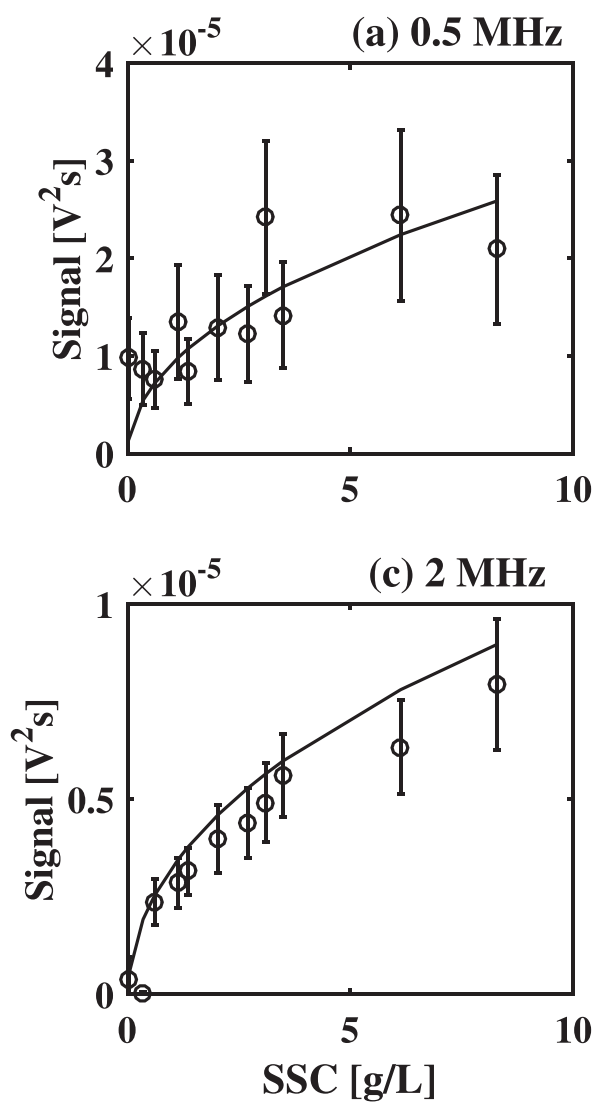
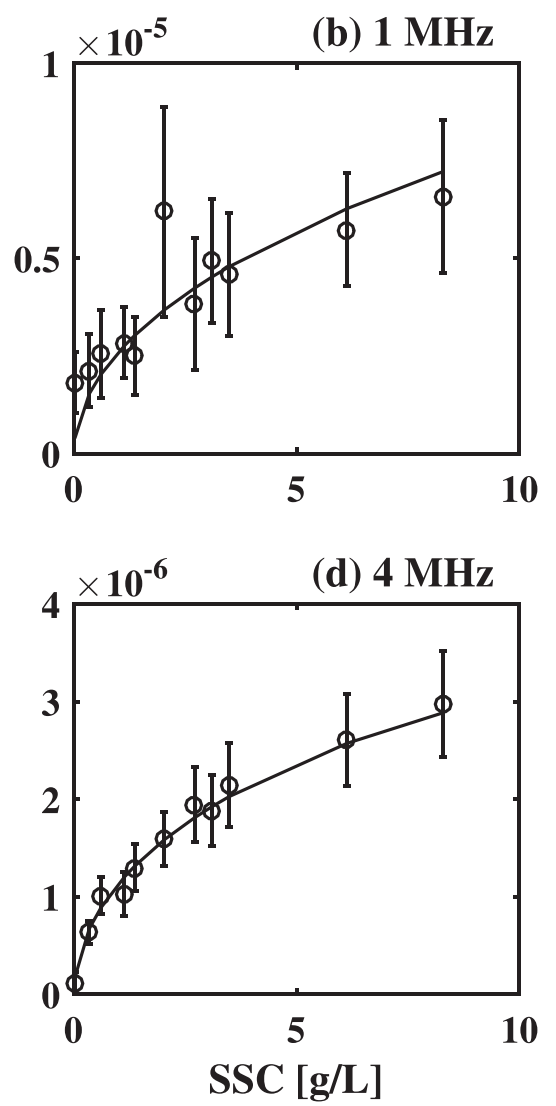

FIG. 9. Experimental backscattering measurements $(\bigcirc)$ and predictions $(-)$ for $20 \mu \mathrm{m}$ size particles at different SSC values for $0.5,1,2$, and $4 \mathrm{MHz}$. Model predictions were done with the combined Faran-AK model. 

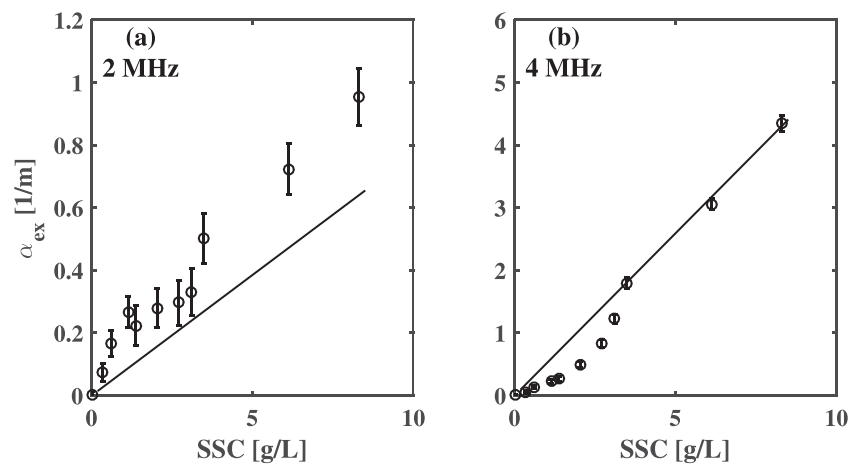

FIG. 10. Experimental (O) and predicted (-) excess attenuation of $105 \mu \mathrm{m}$ size particles at different SSC values for 2 (a) and $4 \mathrm{MHz}$ (b). Model predictions were done with the combined Faran-AK model.

$20 \mu \mathrm{m}$ size particles is shown in Fig. 8. At both frequencies, 2 and $4 \mathrm{MHz}$, the agreement between model predictions and experimental data is satisfactory, definitely good for lower concentrations. Nevertheless, there are some discrepancies for the two largest concentrations, where the excess attenuation is largely overestimated.

The model predictions of the backscattering pressure amplitude could be compared with the signals through the linear correlation between pressure and measured voltage. Since no calibration for the sensors was available, the constants were fitted for each sensor in order to bring model and experiments into agreement. The estimated values of the constants can be found in Table V.

A comparison of the model predictions with the measured backscattering data for $20 \mu \mathrm{m}$ size particles is reported in
Fig. 9. The agreement between model and experiment is satisfactory for 0.5 and $1 \mathrm{MHz}$ considering the large error of the measurements. For 2 and $4 \mathrm{MHz}$ the agreement is quite good.

Turning to the case of $105 \mu \mathrm{m}$ particles the picture is not as clear. The comparison for the attenuation shown in Fig. 10 is still acceptable even though the model underestimates the $2 \mathrm{MHz}$ signal significantly. Nevertheless, the convincing linearity of excess attenuation with concentration seen for the small particles is not visible anymore in this case.

For the $105 \mu \mathrm{m}$ particle backscattering additional deviations from the model can be found (Fig. 11). Here it has to be noted, that the signal quality increases with the measurement frequency. Especially at $4 \mathrm{MHz}$, the model is capturing well the non-monotonous behavior of the signal with increasing concentrations. This non-monotonous behavior is due to both the far field function and the attenuation increasing linearly with the SSC. Since in Eq. (1) the attenuation is in the exponent, at a certain concentration the exponential term is becoming dominant and the backscattered pressure is decreasing.

The difference in signal quality between low and high frequencies and small and large particles can be partially explained for the backscattering measurements by looking at the raw signals (Fig. 12). For all measurements, the backscattering signal follows a small spurious signal resulting from the direct transmission of sound from transducer to receiver. This spurious signal is more significant for 0.5 and $1 \mathrm{MHz}$ and even overlaps to a small extent with the backscattering portion of the signals, while for 2 and $4 \mathrm{MHz}$ the separation is very good. Such behavior is due to the smaller scattering contribution at lower frequencies. Furthermore, the transducer
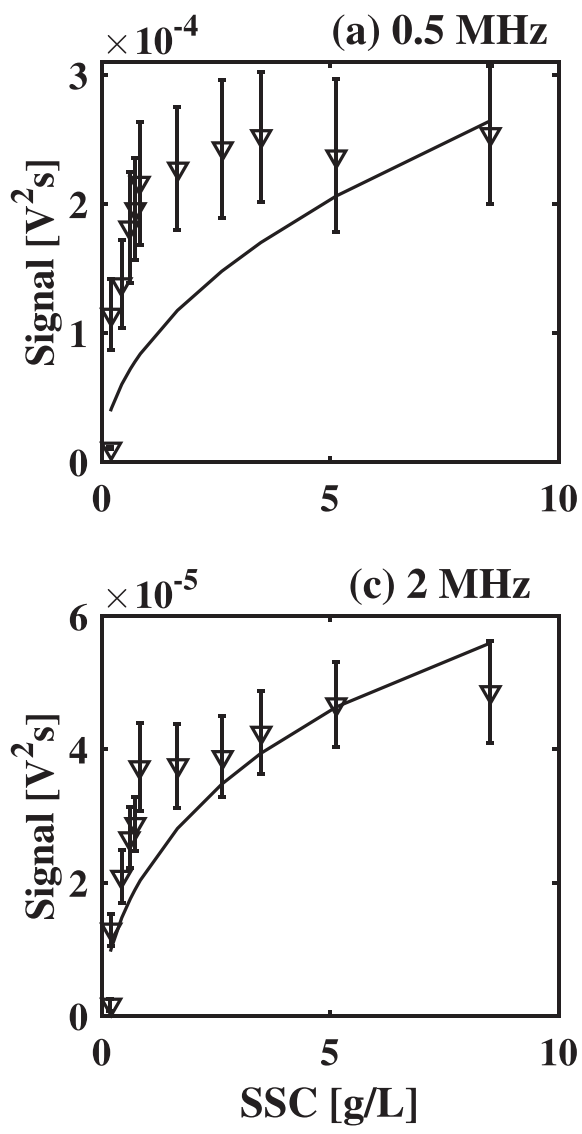
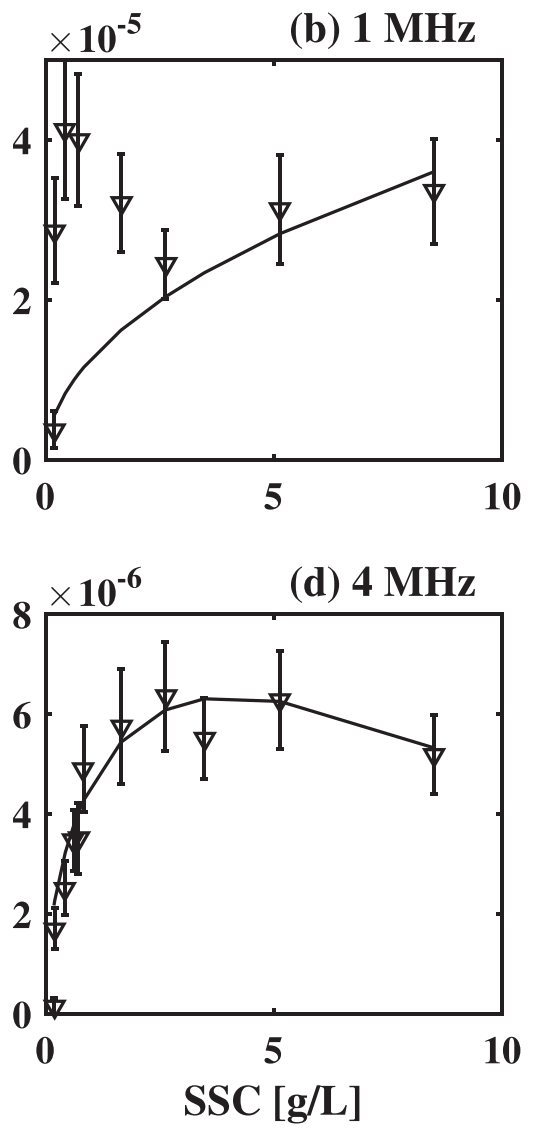

FIG. 11. Experimental backscattering measurements $(\nabla)$ and predictions $(-)$ for $105 \mu \mathrm{m}$ size particles at SSC values for $0.5,1,2$, and $4 \mathrm{MHz}$. Model predictions were done with the combined Faran-AK model. 

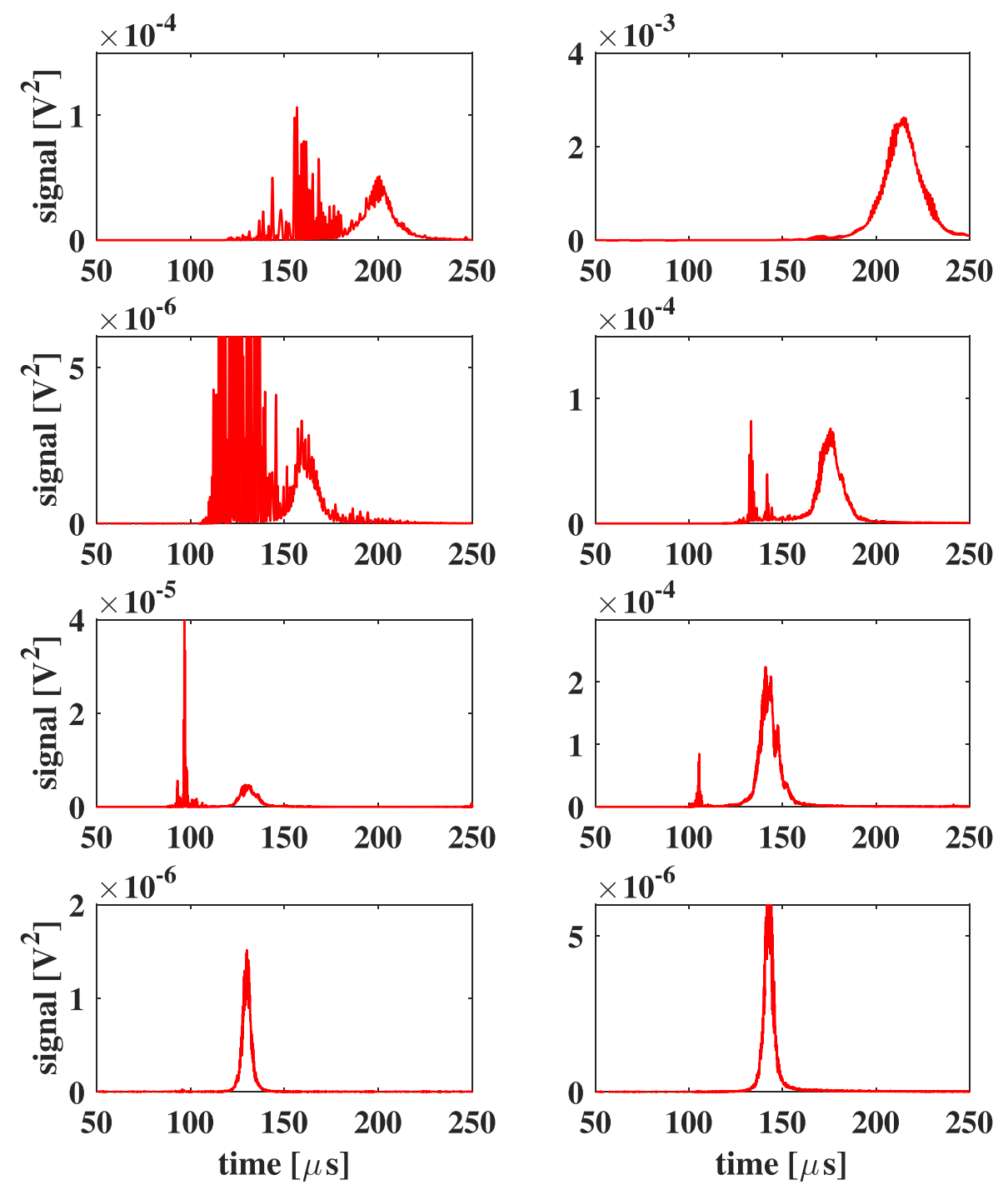

FIG. 12. (Color online) Average squared signals at $8 \mathrm{~g} / \mathrm{L} \mathrm{SSC}$. Top to bottom: 0.5 , 1, 2, and $4 \mathrm{MHz}$. Particle size: $20 \mu \mathrm{m}$ (left), $105 \mu \mathrm{m}$ (right).

function is much narrower at larger frequencies, thus the forward signal at this angle becomes less significant. Finally, the standard deviation of the $105 \mu \mathrm{m}$ particle measurements is larger compared to that of the smaller particles.

\section{Sensitivity analysis}

In the previously discussed experiments, uncertainty can arise from several sources. Starting with the light scattering measurement to determine the particle size distribution over the gravimetric measurement of the concentration to the inherent variability of the ultrasonic signals, the error can be quite significant. A sensitivity analysis was performed on the most significant parameters of the suspension in order to understand their influence on the backscattered signal and excess attenuation. Three parameters-concentration, average particle size, and standard deviation of the PSD-have been reduced and augmented by $10 \%$ and $20 \%$, and the predicted excess attenuations and backscattered signals were plotted in comparison with the results corresponding to the unchanged parameters.

In Fig. 13 the sensitivity of the backscattered signal to the average particle size can be seen. This is the parameter showing the highest sensitivity, with a difference of around
$100 \%$ when changing the parameter by $20 \%$. This influence is very significant. The effect of changing the particle concentration under the same conditions is around $20 \%$, while the effect of a change in the standard deviation is practically negligible.

A similar trend can be seen for the attenuation (Fig. 14). Even in this case the most significant effect is due to a change in the average particle size, where also a $20 \%$ change leads to variation of up to $100 \%$ in the predicted attenuation, as observed for the backscattered intensity. For the $20 \mu \mathrm{m}$ size particles at $4 \mathrm{MHz}$ [Fig. 14(b)], an interesting asymmetry in sensitivity can be seen. The sensitivity is much larger for increasing than decreasing the particle size. This can be explained by examining the attenuation as a function of $k a$ again (Fig. 5): with an average particle size of $20 \mu \mathrm{m}$ and a frequency of $2 \mathrm{MHz}$, the operating $k a$ value is very close to that corresponding to balanced contribution of viscous and scattering dissipations. Increasing the size will move to the scattering regime, while decreasing it will move to the dissipation regime, thus explaining the asymmetric behavior.

This sensitivity analysis shows that an error in the measured signals of about $30 \%$ will lead to an error of about $20 \%$ in the estimated particle size, and even smaller errors in 


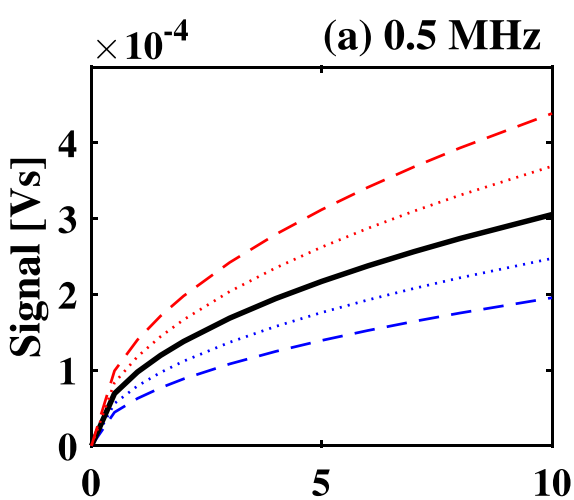

(c) $2 \mathrm{MHz}$

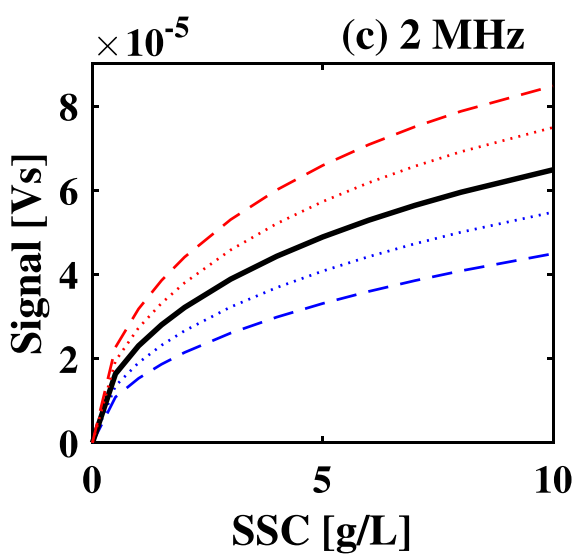

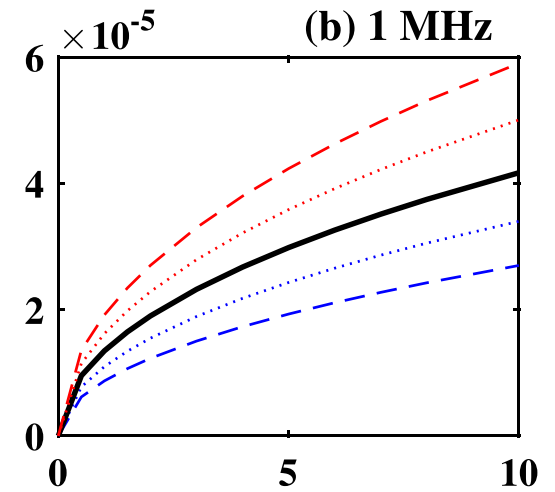

(d) $4 \mathrm{MHz}$

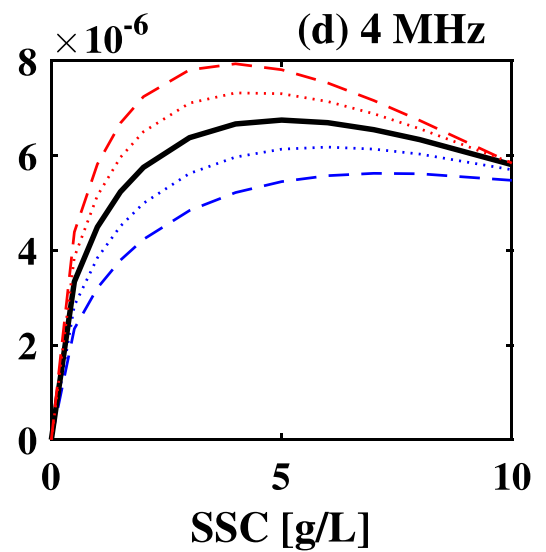

FIG. 13. (Color online) Sensitivity analysis of the predicted backscattered signal on the average particle size. (a) $0.5 \mathrm{MHz}$, (b) $1 \mathrm{MHz}$, (c) $2 \mathrm{MHz}$, (d) $4 \mathrm{MHz}$. Bold lines: predictions for $105 \mu \mathrm{m}$ particles. Fine lines: predictions for $10 \%$ (dotted) and $20 \%$ (dashed) increase (red, above the bold line) and decrease (blue, below the bold line) in average particle size. the estimation of the concentration and polydispersity. These errors are quite acceptable, given the intrinsic limitations and typical uncertainties in experimentally measured ultrasound signals.

\section{CONCLUSIONS}

In this work, backscattering experiments of a suspension of glass beads have been performed in a specially designed tank. Backscattered signals at four different frequencies have been recorded, together with the forward attenuation, measured at two different frequencies. A mathematical model was developed based on a combination of Faran's theory accounting for scattering from the particles and Anderson-Kytömaa's theory to account for viscous dissipation effects. The model validity has first been tested in comparison with the more rigorous ECAH theory, and then used to quantitatively describe the experimental data. The combination of Faran and Kytömaa models was found to be able to well describe the backscattering behavior of glass beads at different sizes. The modeling approach is valid for both the backscattering and the forward attenuation measurements.
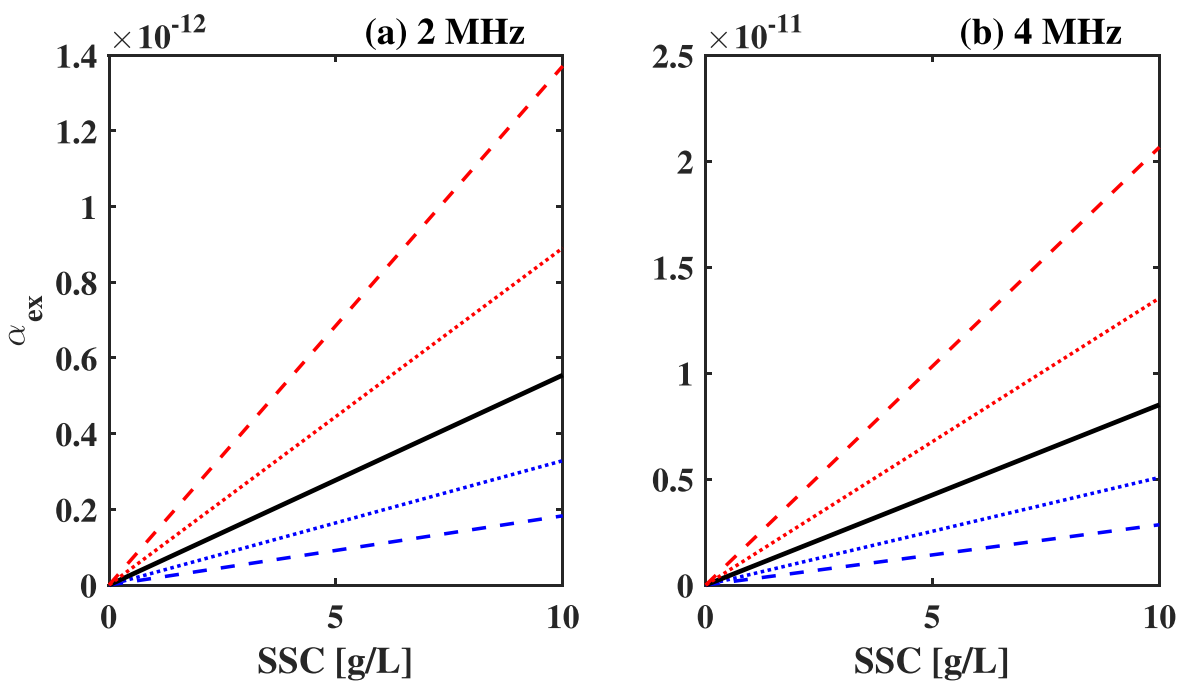

FIG. 14. (Color online) Sensitivity analysis of the predicted excess attenuation on the average particle size. (a) $2 \mathrm{MHz}$, (b) $4 \mathrm{MHz}$. Bold line prediction for $20 \mu \mathrm{m}$ size particles. Fine lines: prediction for $10 \%$ (dotted) and 20\% (dashed) increase (red, above the bold line) and decrease (blue, below the bold line) in average particle size. 
Due to the non-monotonous behavior of the backscattering signals, it appears that reliable estimates of particle size and concentration cannot be carried out by only using one single frequency: at least a few frequencies are needed to extract from the measurements the suspension parameters, provided that their physical properties are well known.

\section{ACKNOWLEDGMENTS}

One of the authors (M.L.) acknowledges financial support from the Swiss National Science Foundation, with Grant No. PP00P2_159258. The project was financially supported by the Commission for Technology and Innovation (CTI), with Grant No. 13223.1 in collaboration with Rittmeyer AG.

\section{LIST OF SYMBOLS}

\author{
A Particle radius \\ A Shear potential \\ c Total sound speed in the suspension \\ $c_{1}$ Compressional sound speed in the particle \\ $c_{2}$ Shear sound velocity in the particle \\ $c_{3}$ Sound velocity in the continuous phase \\ $D_{i} \quad$ Transducer function of transmitter \\ $D_{s} \quad$ Transducer function of receiver \\ $f_{\infty} \quad$ Far field function \\ $h_{n} \quad$ Hyperbolic Bessel function \\ $J_{n} \quad$ Bessel function of the first kind \\ $k$ Complex wavenumber \\ $k_{1}$ Compressional wavenumber in the particle \\ $k_{2} \quad$ Shear wavenumber in the particle \\ $k_{3}$ Compressional wavenumber in the continuous \\ phase \\ $L_{\text {path }}$ Path length from transmitter to receiver \\ $M$ Mass concentration \\ $N_{n} \quad$ Bessel function of the second kind \\ $p^{*} \quad$ Reference pressure \\ $p_{i} \quad$ Incident pressure \\ $p_{s} \quad$ Scattering pressure \\ $P_{n} \quad$ Legendre polynomial \\ $r_{i}, \theta_{i}, \varphi_{i} \quad$ Spherical coordinates \\ $R(t) \quad$ Total path length of signal \\ $r^{*} \quad$ Reference distance \\ $r_{i} \quad$ Incident path length \\ $r_{i, 0} \quad$ Primary axis in the incident path direction \\ $r_{s} \quad$ Scattering path length \\ $r_{s, 0} \quad$ Primary axis in the scattering path direction \\ $t$ Time \\ $u$ Displacement of a sphere by an acoustical wave \\ $V_{\text {exp }} \quad$ Recorded voltage over time of the ultrasonic \\ transducers \\ $x, y, z \quad$ Cartesian coordinates \\ $x_{1}, x_{2}, x_{3} \quad k_{1} a, k_{2} a, k_{3} a$ \\ $\alpha$ Total attenuation \\ $\alpha_{\text {ex }}$ Excess attenuation \\ $\alpha_{1} \quad$ Attenuation of the mixture \\ $\alpha_{n}, \beta_{n}, \delta_{n} \quad$ Intermediate angles
}

$\alpha_{s} \quad$ Attenuation of the sediment

$\begin{aligned} \beta_{0}, \beta_{i}, \beta_{s} & \text { Transducer angles } \\ \delta & \text { Boundary layer thickness } \\ \Delta R & \text { Signal length } \\ \eta_{n} & \text { Phase shift of } n \text {th scattered wave } \\ \kappa_{s} & \text { Bulk modulus of solid phase } \\ \kappa_{l} & \text { Bulk modulus of continuous phase } \\ \bar{\kappa} & \text { Average bulk modulus } \\ \mu_{1} & \text { Viscosity liquid phase } \\ \rho *, \rho & \text { Average densities } \\ \rho_{l} & \text { Density of the liquid phase } \\ \rho_{s} & \text { Density of the solid phase } \\ \sigma & \text { Poisson ratio of the solid phase } \\ \varphi & \text { Volume fraction } \\ \Phi_{n} & \text { Boundary impedance phase angle } \\ \psi & \text { Compressional potential } \\ \omega & \text { Angular frequency }\end{aligned}$

\section{APPENDIX A: MODEL DERIVATION}

\section{Faran model}

The Faran model for the scattering of sound waves by spherical particles is based on three main assumptions: (i) the mechanical behavior of the particle follows classical continuous mechanics for elastic objects, (ii) the continuous phase is considered inviscid, and (iii) thermal dissipation is neglected. Additional assumptions are plane incident sound wave, steady state irradiation and wavelength larger than the particle size. The displacement $u$ of a sphere by an acoustical wave can then be derived from a scalar potential, $\psi$ and a vector potential $A$,

$$
u=-\nabla \psi+\nabla \times \mathbf{A}
$$

The displacement can be separated into the two wave equations

$$
\begin{aligned}
& \nabla^{2} \psi=\frac{1}{c_{1}^{2}} \frac{\partial^{2} \psi}{\partial t^{2}} \\
& \nabla^{2} \mathbf{A}=\frac{1}{c_{2}^{2}} \frac{\partial^{2} \mathbf{A}}{\partial t^{2}} .
\end{aligned}
$$

Equations (A2) and (A3) describe the propagation of longitudinal and shear waves, respectively. The solution of these equations requires the specification of three boundary conditions at the particle interface: (i) the pressure of the fluid and normal stress are equal, (ii) the displacement of fluid and solid is equal, and (iii) the tangential and shear stresses are zero. The relationship between displacement and pressure is given by

$$
u_{r}=\frac{1}{\rho_{l} \omega^{2}} \frac{\partial p_{i}}{\partial r} .
$$

Accordingly, the scattered pressure becomes 


$$
\begin{aligned}
p_{s}= & p_{i} \sum_{n=0}^{\infty}(2 n+1)(-1)^{n} \sin \left(\eta_{n}\right) \\
& \times \exp \left(-i \eta_{n}\right) h_{n}\left(k_{c} r\right) P_{n}(\cos \theta),
\end{aligned}
$$

where $p_{i}$ is the incident pressure, $\theta$ is the scattering angle and $h_{n}$ is the hyperbolic Bessel function. The so-called phase shift of the $n$th scattered wave $\eta_{n}$ is defined as

$$
\tan \eta_{n}=\tan \left(\delta_{n}\left(x_{3}\right)\right) \frac{\tan \Phi_{n}+\tan \left(\alpha_{n}\left(x_{3}\right)\right)}{\tan \Phi_{n}+\tan \left(\beta_{n}\left(x_{3}\right)\right)},
$$

with the intermediate angles given by

$$
\begin{aligned}
& \delta_{n}\left(x_{i}\right)=\tan ^{-1}\left(\frac{-J_{n}\left(x_{i}\right)}{N_{n}\left(x_{i}\right)}\right), \alpha_{n}\left(x_{i}\right)=\tan ^{-1}\left(\frac{x_{i} J_{n}^{\prime}\left(x_{i}\right)}{J_{n}\left(x_{i}\right)}\right), \\
& \beta_{n}\left(x_{i}\right)=\tan ^{-1}\left(\frac{x_{i} N^{\prime}{ }_{n}\left(x_{i}\right)}{N_{n}\left(x_{i}\right)}\right),
\end{aligned}
$$

where $J_{n}$ and $N_{n}$ are Bessel functions of the first and second kind, respectively. The boundary impedance phase angle $\Phi_{n}$ is equal to

$$
\Phi_{n}=-\frac{\rho_{l}}{\rho_{s}} \frac{x_{2}^{2}}{2} \frac{\frac{x_{1} J^{\prime}{ }_{n}\left(x_{1}\right)}{x_{1} J^{\prime}\left(x_{1}\right)-J_{n}\left(x_{1}\right)}-\frac{2\left(n^{2}+n\right) J_{n}\left(x_{2}\right)}{\left(n^{2}+n-2\right) J_{n}\left(x_{2}\right)+x_{2}^{2} J^{\prime \prime}{ }_{n}\left(x_{2}\right)}}{\frac{x_{n}^{2}\left\{[\sigma /(1-2 \sigma)] J_{n}\left(x_{1}\right)+J^{\prime \prime}{ }_{n}\left(x_{1}\right)\right\}}{x_{1} J^{\prime}{ }_{n}\left(x_{1}\right)-J_{n}\left(x_{1}\right)}-\frac{2\left(n^{2}+n\right)\left[J_{n}\left(x_{2}\right)+x_{2} J^{\prime}{ }_{n}\left(x_{2}\right)\right]}{\left(n^{2}+n-2\right) J_{n}\left(x_{2}\right)+x_{2}^{2} J^{\prime \prime}{ }_{n}\left(x_{2}\right)}},
$$

where the function $\Phi_{n}$ is easy to implement and numerically stable. Furthermore, it only depends on three physical properties of the solid: the density $\rho_{s}$, the compressional velocity $c_{1}$, and the Poisson ratio $\sigma$. The last two quantities are involved in the expressions of $x_{i}$ as follows:

$$
\begin{aligned}
& x_{1}=k_{1} a=\frac{\omega}{c_{1}} a, \\
& x_{2}=k_{2} a=\frac{\omega}{c_{2}} a, \\
& x_{3}=k_{c} a=\frac{\omega}{c} a,
\end{aligned}
$$

where $a$ is the particle radius and $c_{2}$ is the shear velocity, which is given as a function of $c_{1}$ and $\sigma$. Note that in this work we use $k_{c}$, and $\mathrm{c}$ for the wavenumber and velocity of sound in the liquid, while in the original work of Faran these variables are called $k_{3}$ and $c$,

$$
c_{2}=\sqrt{\frac{3 c_{1}^{2}(1-2 \sigma)}{2(3-5 \sigma)}} .
$$

Using these quantities, one can calculate the far field function $f_{\infty}$, defined as an approximation of the amplitude of a scattered wave at large distance from the particle,

$$
f_{\infty}=\frac{2 r}{a} \frac{p_{s}}{p_{i}}
$$

Equation (A5), the equation for the pressure, can be simplified at large distances from the sphere as follows:

$$
\lim _{r \rightarrow \infty}\left|p_{s}\right|=\frac{p_{i}}{k_{c} r}\left|\sum_{n=0}^{\infty} i(2 n+1) \sin \left(\eta_{n}\right) \exp \left(-i \eta_{n}\right) P_{n}(\cos \theta)\right|
$$

where $r$ is the distance from the point scatterer. This leads to the following expression for the far field function for the Faran model:

$$
f_{\infty}=-\frac{2}{x_{3}} \sum_{n=0}^{\infty} i(2 n+1) \sin \left(\eta_{n}\right) \exp \left(-i \eta_{n}\right) P_{n}(\cos \theta)
$$

The attenuation coefficient $\alpha_{s}$ describes the loss of acoustic energy over a distance $\Delta z=z_{1}-z_{2}$, and can be expressed as

$$
\alpha_{s}=\frac{1}{\Delta z} \ln \frac{\left.p\right|_{z_{1}}}{\left.p\right|_{z_{2}}}
$$

where $p$ is the pressure at location $z_{1}$ or $z_{2}$, respectively. An expression for the attenuation for the ECAH model can be found in the literature. ${ }^{7,8}$ Using similarities in the ECAH and Faran models, the following expression for the attenuation in the Faran model was found:

$$
\alpha_{s}=\frac{-3}{2\left(k_{c} a\right)^{2} a} \sum_{n=0}^{\infty}(2 n+1) \Re e\left\{-i \cdot \sin \left(\eta_{n}\right) \exp \left(-i \eta_{n}\right)\right\}
$$

Note that the attenuation has to be multiplied by the volume fraction of particles and added to the attenuation of the dispersed phase.

\section{Atkinson-Kytömaa model}

In the semi empirical approach of Atkinson and Kytömaa, the acoustic wave propagation is described by the complex wave number $k$, which is a parameter involving both sound speed, $c$, and wave attenuation, $\alpha$, 


$$
k=\frac{\omega}{c}+i \alpha
$$

where $\omega$ is the angular frequency of the sound wave. According to the literature, ${ }^{67}$ three different regimes can be identified depending on the ratio between the radius $a$ of the suspended particles and the wavelength $\lambda$ of the propagating wave: a SWR $(a>\lambda)$, an intermediate wavelength regime $(a$ $\approx \lambda$ ), and a LWR $(a<\lambda)$. For each regime, different mechanisms of liquid-particle interaction have to be accounted for. MATHEMATICAL models for evaluating the wave number in each of the different regimes are available. 9,67,68 The AK model was originally proposed for suspensions of monodisperse spherical particles oscillating with frequency $f$ in a stationary Newtonian liquid of viscosity $\mu_{1}$, with particle volume fraction $\varphi$. The liquid $(l)$ and solid $(s)$ phases are both described as a continuum. Each phase $i$ is characterized by density, $\rho_{i}$, and bulk modulus, $\kappa_{i}$. In the AK model, all viscous dissipations are confined into a thin boundary layer surrounding the particles, whose thickness is given by

$$
\delta=\sqrt{\frac{2 \mu_{l}}{\rho_{l} \omega}} .
$$

For this reason, the attenuation given by the AK model is the excess attenuation only, namely, the total attenuation subtracted by the intrinsic attenuation of the mixture, $\alpha_{I}$,

$$
\alpha_{e x}=\alpha-\alpha_{I}=\alpha-\sum_{i} \varphi_{i} \alpha_{i}
$$

where $\alpha_{i}$ and $\varphi_{i}$ are intrinsic attenuation and volume fraction of each pure material, respectively. The wave number in the AK model is expressed as

$$
k^{2}=\frac{\omega^{2}}{\bar{\kappa}} \frac{\left[(A+i \omega B) \bar{\rho}+i \omega \rho_{s} \rho_{l}(1-\varphi)\right]}{A+i \omega B+i \omega \rho^{*}(1-\varphi)},
$$

where average densities and bulk moduli are evaluated as

$$
\begin{aligned}
& \bar{\kappa}=\left(\frac{\varphi}{\kappa_{s}}+\frac{(1-\varphi)}{\kappa_{l}}\right)^{-1}, \\
& \rho^{*}=(1-\varphi) \rho_{s}+\varphi \rho_{l}, \\
& \bar{\rho}=\varphi \rho_{s}+(1-\varphi) \rho_{l},
\end{aligned}
$$

and the quantities $A$ and $B$ are expressed as

$$
\begin{aligned}
& A=\frac{9 \mu}{2 a^{2}}\left(1+\frac{a}{\delta}\right), \\
& B=\rho_{l}\left[\left(\frac{1-\varphi}{2}\right)+\frac{9 \mu}{4 a}\right] .
\end{aligned}
$$

Given the value of $\omega$ (which is the case in applications), both $\varphi$ (volume fraction of the dispersed phase) and $r$ can be estimated by measuring both $c$ and $\alpha_{e x}$. In contrast, if the particle size is given, $\varphi$ can be estimated by measuring one variable only, $c$ or $\alpha_{e x}$. In both cases, accurate values of the pure materials properties $\left[\rho_{i}\right.$ and $\kappa_{i}$ or, equivalently, $\rho_{i}$ and $\left.c_{i}=\left(\kappa_{i} / \rho_{i}\right)^{1 / 2}\right]$, must be available.

\section{APPENDIX B: ANGULAR BACKSCATTERING FROM A} SUSPENSION

Considering a monodisperse suspension of spherical particles with radius $a$, density $\rho_{s}$, and mass concentration $M$, the number concentration of the particles is given by

$$
N=\frac{M}{(4 / 3) \pi a^{3} \rho_{s}} .
$$

The backscattered pressure from the infinitesimal point scatterer located at $\left(r_{i}, \theta_{i}, \varphi_{i}\right)$ is given by ${ }^{61}$

$$
\left|p_{s}\right|=\frac{M}{(4 / 3) \pi a^{3} \rho_{s}} \frac{p^{*} r^{*}}{r_{i} r_{s}} D_{i} D_{s} f_{\infty}(\theta, a) a \exp \left(-\alpha\left(r_{i}+r_{s}\right)\right),
$$

where $\alpha$ and $f_{\infty}$ are defined in the Appendix A, $r_{i}$ is the incident path length from the transmitter to the point of the scattering event, and $r_{s}$ is the scatter path length from the point of the scattering event to the receiver. The scattering angle $\theta$ is defined through the position of transmitter, receiver, and point scatterer. $D_{i}$ and $D_{s}$ are the transducer functions defining the intensity of the incident pressure and sensitivity to signals reaching the receiver, respectively. They are functions of the transducer membrane radius $a_{t}$, the wavenumber $k$, and the angle relative to the principal axis of the transducer, $\beta$. For the incident transducer, as an example, the function can be calculated through the following formula [with $J_{l}(x)$ being the Bessel function of order 1]:

$$
D_{i}=2 \frac{J_{1}\left(k_{c} a_{t} \sin \left(\beta_{i}\right)\right)}{k_{c} a_{t} \sin \left(\beta_{i}\right)} .
$$

In a recent publication, ${ }^{69}$ a model for the transducer function has been proposed in the form of a Gaussian distribution and experimentally tested. Accordingly, in this work the transducer function has been approximated in the same way, using a standard deviation, $\sigma$, dependent on the transducer frequency,

$$
D_{g, i}=e^{\left(-\beta_{i}^{2} / 2 \sigma^{2}\right)}
$$

The overlap of the $4 \mathrm{MHz}$ transducer function [Eq. (B3)] and the corresponding Gaussian approximation [Eq. (B4)] is shown in Fig. 15: even though the minor peaks are not reproduced by the approximate distribution, the main peak at $\beta=0$ is well captured. Therefore, in this work the approximation was used, since it leads to less numerical instabilities in the integration over the volume described in this section. The real membrane diameters of the transducers used in this work and the corresponding standard deviation of the approximating Gaussian functions are summarized in Table VI.

One assumption for the measurement is that the transmitter emits a step pulse with duration $\tau$, frequency $f$, and reference pressure $p^{*}$ at reference distance $r^{*}$ from the transmitter. To obtain the backscattered pressure, one has to integrate the pressure over all points from which the sound waves reach the receiver at the same time $t$. These points are all located between the surface of two spheroids which have the location of transmitter and receiver as foci. The surfaces are then given by the conditions 


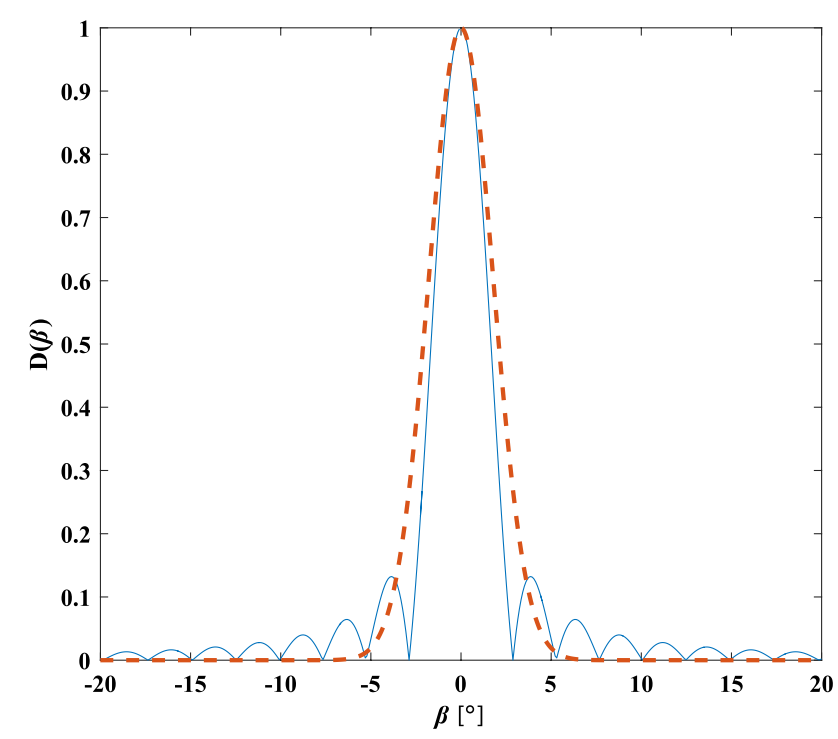

FIG. 15. (Color online) Transducer function [Eq. (B3)] for the $0.5 \mathrm{MHz}$ transducer (solid blue curve) and corresponding Gaussian approximation [Eq. (B4), dashed red curve].

$$
R<r_{i}+r_{s}<R+\Delta R
$$

where $R=c t$ and $\Delta R=c \tau, r_{i}$ is the incident path length, and $r_{s}$ the path length of the scattered wave.

The setup of transmitter and receiver is schematically shown in Fig. 16. Their principal axes are in the same plane, where $\beta_{0}$ is the angle in between. The setup is described by the equation

$$
r_{i 0}\left(\begin{array}{l}
1 \\
0 \\
0
\end{array}\right)+r_{s 0}\left(\begin{array}{c}
x_{0} \\
y_{0} \\
0
\end{array}\right)=r_{0}\left(\begin{array}{c}
\cos \beta_{0} \\
\sin \beta_{0} \\
0
\end{array}\right),
$$

where $x_{0}$ and $y_{0}$ define the direction of the primary scattering path and $r_{0}$ defines the distance of the direct path from transmitter to receiver. This equation can be solved to obtain $x_{0}$ and $y_{0}$.

To integrate the signal over a given finite volume element, it is necessary to calculate the scattering angle $\theta$, the incident path length $r_{i}$, the scattering path length $r_{s}$, the angle between the principal axis of transmitter and the incident path angle $\beta$, and the angle between the principal axis of the receiver and the scattering path $\beta^{\prime}$. The principal axis of the receiver originates at $r=0$ into the direction $\theta=0$ and $\varphi=0$. The incident path angle $\beta_{i}$ is then given by

$$
\cos \beta_{i}=\cos \theta_{i} \cos \varphi_{i} \text {. }
$$

For the triangle formed by an arbitrary point in space, transmitter and receiver, a condition similar to Eq. (B5) can be set up,

TABLE VI. Parameter values of the transducer function and its approximation.

\begin{tabular}{lcccc}
\hline \hline Transducer frequency & $0.5 \mathrm{MHz}$ & $1 \mathrm{MHz}$ & $2 \mathrm{MHz}$ & $4 \mathrm{MHz}$ \\
\hline$a_{t}$ & 36 & 20 & 11.2 & 8.6 \\
$\sigma$ & 0.0310 & 0.0281 & 0.0251 & 0.0129 \\
\hline \hline
\end{tabular}

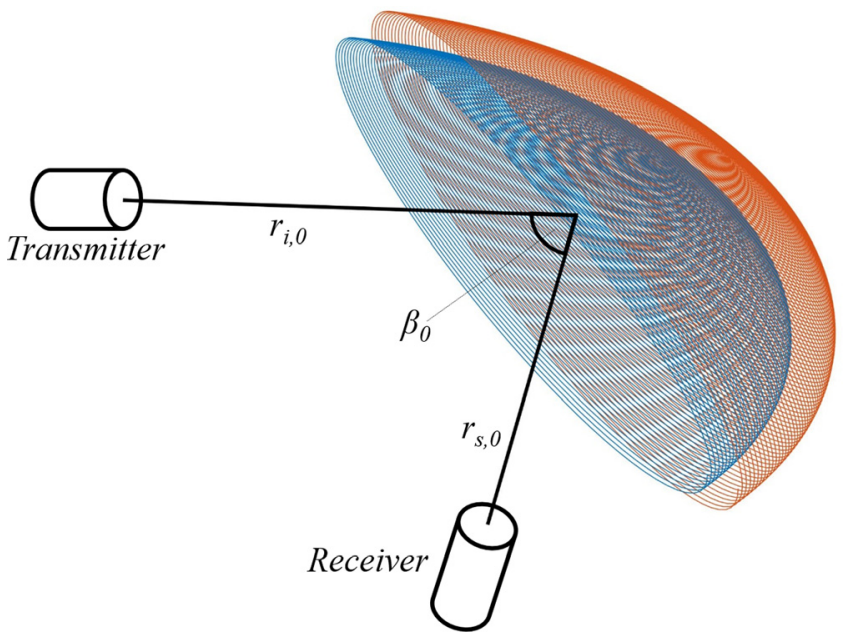

FIG. 16. (Color online) The two shaded surfaces confine a volume element from which the sound is reflected to the receiver at a given time $\mathrm{t}$.

$$
\begin{aligned}
& r_{i}\left(\begin{array}{c}
\cos \varphi_{i} \cos \theta_{i} \\
\cos \varphi_{i} \sin \theta_{i} \\
\sin \varphi_{i}
\end{array}\right)+r_{s}\left(\begin{array}{c}
\cos \varphi_{s} \cos \theta_{s} \\
\cos \varphi_{s} \sin \theta_{s} \\
\sin \varphi_{s}
\end{array}\right) \\
& =r_{0}\left(\begin{array}{c}
\cos \beta_{0} \\
\sin \beta_{0} \\
0
\end{array}\right) .
\end{aligned}
$$

Given $R=r_{i}+r_{s}$, the equation can be written as

$$
\begin{aligned}
& r_{i}\left(\begin{array}{c}
\cos \varphi_{i} \cos \theta_{i} \\
\cos \varphi_{i} \sin \theta_{i} \\
\sin \varphi_{i}
\end{array}\right)+\left(R-r_{i}\right)\left(\begin{array}{c}
\cos \varphi_{s} \cos \theta_{s} \\
\cos \varphi_{s} \sin \theta_{s} \\
\sin \varphi_{s}
\end{array}\right) \\
& \quad=r_{0}\left(\begin{array}{c}
\cos \beta_{0} \\
\sin \beta_{0} \\
0
\end{array}\right) .
\end{aligned}
$$

These are three equations with the three unknowns $r_{i}, \varphi_{s}, \theta_{s}$, which can be solved for $r_{i}$,

$$
r_{i}=\frac{r_{0}^{2}+R^{2}}{-2 r_{0} \cos \varphi_{i}\left(\sin \beta_{0} \sin \theta_{i}+\cos \beta_{0} \cos \theta_{i}\right)+R} .
$$

Given $r_{i}, \varphi_{s}$, and $\theta_{s}$ one can easily calculate $r_{s}$. The scattering angle $\theta$ is then given by the product of the two vectors $r_{i}$ and $r_{s}$,

$$
\cos \theta=\frac{r_{i}\left(\begin{array}{c}
\cos \varphi_{i} \cos \theta_{i} \\
\cos \varphi_{i} \sin \theta_{i} \\
r \sin \varphi_{i}
\end{array}\right) r_{s}\left(\begin{array}{c}
\cos \varphi_{s} \cos \theta_{s} \\
\cos \varphi_{s} \sin \theta_{s} \\
\sin \varphi_{s}
\end{array}\right)}{r_{i} r_{s}} .
$$

And finally $\beta_{s}$ is given by

$$
\cos \beta_{s}=\frac{r_{s}\left(\begin{array}{c}
\cos \varphi_{s} \cos \theta_{s} \\
\cos \varphi_{s} \sin \theta_{s} \\
\sin \varphi_{s}
\end{array}\right) r_{s 0}\left(\begin{array}{c}
x_{0} \\
y_{0} \\
0
\end{array}\right)}{r_{s} r_{s 0}} .
$$

For the measurement of sediments, an acoustic pulse of duration $\tau$ is sent into the suspension. As already mentioned, the backscattered signal is integrated between the ellipsoidal volume fraction defined by 


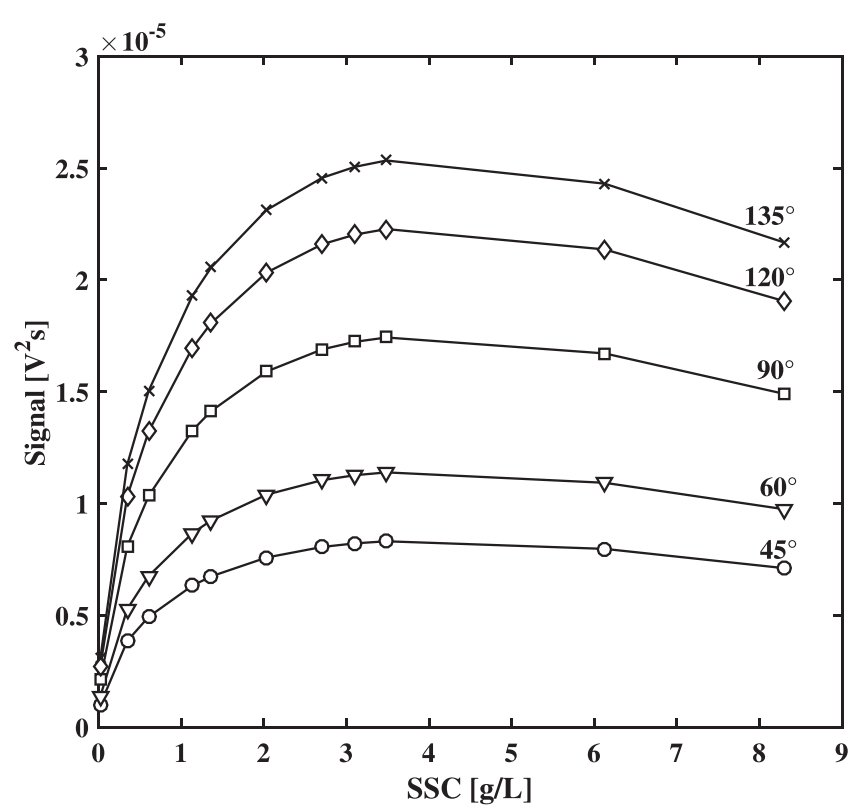

FIG. 17. An example for a predicted backscattering signal at different angles for $105 \mu \mathrm{m}$ particles.

$$
R+\Delta R=c t+c \tau
$$

By integrating over these variables as well as the azimuthal and equatorial angles, $\psi^{*}$ and $\theta^{*}$, the following expression for the backscattered pressure at a given time is finally obtained:

$$
\begin{aligned}
\left|p_{s}\right|^{2}= & \frac{3 M p^{* 2} r^{* 2}}{4 \pi\left\langle a^{3}\right\rangle \rho_{s}} \\
& \times \int_{-\pi}^{\pi} \int_{-\pi / 2}^{\pi / 2} \int_{R}^{R+\Delta R}\left[\frac{\left\langle f_{\infty}(\theta, a) a\right\rangle^{2}}{\left(r_{i} r_{s}\right)^{2}}\left(D_{i} D_{s}\right)^{2}\right. \\
& \left.\times \exp \left(-2\langle\alpha\rangle\left(r_{i}+r_{s}\right)\right) r_{i}^{2} \frac{\partial r_{i}}{\partial R} \cos \varphi_{i}\right] d r_{i} d \varphi_{i} d \theta_{i}
\end{aligned}
$$

where the spatial derivative is given by

$$
\frac{\partial r_{i}}{\partial R}=\frac{R^{2}-4 R r_{0} \cos \varphi_{i}\left(\sin \beta_{0} \sin \theta_{i}+\cos \beta_{0} \cos \theta_{i}\right)-r_{0}^{2}}{\left(-2 r_{0} \cos \varphi_{i}\left(\sin \beta_{0} \sin \theta_{i}+\cos \beta_{0} \cos \theta_{i}\right)+R\right)^{2}} .
$$

Using Eq. (B14), one can predict the backscattered pressure from any given homogenous suspension of particles with defined particle size distribution, concentration, using any given angle or distance between transmitter and receiver. Figure 17 shows an example of backscattering at different angles. In the range between $45^{\circ}$ and $135^{\circ}$ there are quantitative changes, even though the qualitative behavior stays the same. Going for smaller angles would mean going into the direct backscattering, while going for higher angles the signal would superimpose more and more with the direct signal.

${ }^{1}$ A. K. Hipp, G. Storti, and M. Morbidelli, "Acoustic characterization of concentrated suspensions and emulsions. 1. Model analysis," Langmuir 18, 391-404 (2002).
${ }^{2}$ E. Müller and C. Mann, "Resin characterization by electro-acoustic measurements," J. Chromatogr. A 1144, 30-39 (2007).

${ }^{3}$ A. K. Hipp, B. Walker, M. Mazzotti, and M. Morbidelli, "In-situ monitoring of batch crystallization by ultrasound spectroscopy," Ind. Eng. Chem. Res. 39, 783-789 (2000).

${ }^{4}$ F. Scheffold and P. Schurtenberger, "Light scattering probes of viscoelastic fluids and solids," Soft Mater. 1, 139-165 (2003).

${ }^{5}$ A. K. Hipp, G. Storti, and M. Morbidelli, "Acoustic characterization of concentrated suspensions and emulsions. 2. Experimental validation," Langmuir 18, 405-412 (2002).

${ }^{6}$ T. J. Shamu, R. Kotze, and J. Wiklund, "Characterization of acoustic beam propagation through high-grade stainless steel pipes for improved pulsed ultrasound velocimetry measurements in complex industrial fluids," IEEE Sens. J. 16, 5636-5647 (2016).

${ }^{7}$ P. S. Epstein and R. R. Carhart, "The absorption of sound in suspensions and emulsions. I. Water fog in air," J. Acoust. Soc. Am. 25, 553-556 (1953).

${ }^{8}$ J. R. Allegra and S. A. Hawley, "Attenuation of sound in suspensions and emulsions: Theory and experiments," J. Acoust. Soc. Am. 51, 1545-1564 (1972).

${ }^{9}$ C. M. Atkinson and H. K. Kytomäa, "Acoustic wave speed and attenuation in suspensions,” Int. J. Multiph. Flow 18, 577-592 (1992).

${ }^{10}$ C. M. Atkinson and H. K. Kytomäa, "Acoustic properties of solid-liquid mixtures and the limits of ultrasound diagnostics-I: Experiments (data bank contribution)," J. Fluids Eng. 115, 665 (1993).

${ }^{11}$ R. J. Urick, "Theoretical and observed absorption of sound in suspensions," J. Acoust. Soc. Am. 20, 225 (1948).

${ }^{12}$ R. J. Urick, "The absorption of sound in suspensions of irregular particles," J. Acoust. Soc. Am. 20, 283-289 (1948).

${ }^{13}$ A. E. Hay and D. G. Mercer, "On the theory of sound scattering and viscous absorption in aqueous suspensions at medium and short wavelengths," J. Acoust. Soc. Am. 78, 1761-1771 (1985).

${ }^{14}$ J. J. Faran, "Sound scattering by solid cylinders and spheres," J. Acoust. Soc. Am. 23, 405-418 (1951).

${ }^{15}$ W. R. Geyer and R. Signell, "Measurements of tidal flow around a headland with a shipboard acoustic Doppler current profiler," J. Geophys. Res. 95, 3189-3197, https://doi.org/10.1029/JC095iC03p03189 (1990).

${ }^{16}$ C. D. Rennie, R. G. Millar, and M. A. Church, "Measurement of bed load velocity using an acoustic doppler current profiler," J. Hydraul. Eng. 128, 473-483 (2002).

${ }^{17}$ A. Sottolichio, D. Hurther, N. Gratiot, and P. Bretel, “Acoustic turbulence measurements of near-bed suspended sediment dynamics in highly turbid waters of a macrotidal estuary," Cont. Shelf Res. 31, S36-S49 (2011).

${ }^{18}$ R. F. van Unen, P. D. Thorne, H. Cox, and S. D. Kamminga, "Laboratory measurements of current flow using cross correlation on acoustic backscattering from suspended sediments," J. Acoust. Soc. Am. 104, 1345-1355 (1998).

${ }^{19} \mathrm{P}$. D. Thorne and D. Hurther, "An overview on the use of backscattered sound for measuring suspended particle size and concentration profiles in non-cohesive inorganic sediment transport studies," Cont. Shelf Res. 73, 97-118 (2014).

${ }^{20}$ K. F. E. Betteridge, P. D. Thorne, and R. D. Cooke, "Calibrating multifrequency acoustic backscatter systems for studying near-bed suspended sediment transport processes," Cont. Shelf Res. 28, 227-235 (2008).

${ }^{21}$ H. K. Ha, J. P. Y. Maa, K. Park, and Y. H. Kim, "Estimation of highresolution sediment concentration profiles in bottom boundary layer using pulse-coherent acoustic Doppler current profilers," Mar. Geol. 279, 199-209 (2011).

${ }^{22}$ P. D. Thorne, C. E. Vincent, P. J. Hardcastle, S. Rehman, and N. Pearson, "Measuring suspended sediment concentrations using acoustic backscatter devices," Mar. Geol. 98, 7-16 (1991).

${ }^{23}$ P. D. Thorne and P. J. Hardcastle, "Acoustic measurements of suspended sediments in turbulent currents and comparison with in-situ samples," J. Acoust. Soc. Am. 101, 2603-2614 (1997).

${ }^{24}$ G. P. Holdaway, P. D. Thorne, D. Flatt, S. E. Jones, and D. Prandle, "Comparison between ADCP and transmissometer measurements of suspended sediment concentration," Cont. Shelf Res. 19, 421-441 (1999).

${ }^{25}$ K. F. E. Betteridge, P. D. Thorne, and P. S. Bell, "Assessment of acoustic coherent Doppler and cross-correlation techniques for measuring near-bed velocity and suspended sediment profiles in the marine environment," J. Atmos. Ocean. Technol. 19, 367-380 (2002).

${ }^{26}$ J. F. Lynch, J. D. Irish, C. R. Sherwood, and Y. C. Agrawal, "Determining suspended sediment particle size information from acoustical and optical backscatter measurements," Cont. Shelf Res. 14, 1139-1165 (1994). 
${ }^{27}$ C. Shen and U. Lemmin, "Ultrasonic measurements of suspended sediments: A concentration profiling system with attenuation compensation," Meas. Sci. Technol. 7, 1191-1194 (1996).

${ }^{28} \mathrm{M}$. O. Green and K. P. Black, "Suspended-sediment reference concentration under waves: Field observations and critical analysis of two predictive models," Coast. Eng. 38, 115-141 (1999).

${ }^{29}$ T. N. Hunter, L. Darlison, J. Peakall, and S. Biggs, "Using a multifrequency acoustic backscatter system as an in situ high concentration dispersion monitor," Chem. Eng. Sci. 44, 409-418 (2012).

${ }^{30}$ W. O. Carpenter, B. T. Goodwiller, J. P. Chambers, D. G. Wren, and R. A. Kuhnle, "Acoustic measurement of suspensions of clay and silt particles using single frequency attenuation and backscatter," Appl. Acoust. 85, 123-129 (2014).

${ }^{31}$ J. G. Venditti, M. A. Church, M. E. Attard, and D. Haught, "Use of ADCPs for suspended sediment transport monitoring: An empirical approach," Water Resour. Res. 52, 2715-2736, https://doi.org/10.1002/ 2015WR017348 (2016).

${ }^{32}$ C. E. Vincent, D. M. Hanes, and A. J. Bowen, "Acoustic measurements of suspended sand on the shore face and the control of concentration by bed roughness," Mar. Geol. 96, 1-18 (1991).

${ }^{33}$ R. Meral, "Laboratory evaluation of acoustic backscatter and LISST methods for measurements of suspended sediments," Sensors 8, 979-993 (2008).

${ }^{34}$ M. J. W. Povey, Ultrasonic Techniques for Fluids Characterization (Academic, San Diego, 1997).

${ }^{35}$ P. D. Thorne and D. M. Hanes, "A review of acoustic measurement of small-scale sediment processes," Cont. Shelf Res. 22, 603-632 (2002).

${ }^{36}$ P. D. Thorne, P. J. Hardcastle, D. Flatt, and J. D. Humphery, "On the use of acoustics for measuring shallow water suspended sediment processes," IEEE J. Ocean. Eng. 19, 48-57 (1994).

${ }^{37}$ P. H. Wiebe, T. K. Stanton, M. C. Benfield, D. G. Mountain, and C. H. Greene, "High-frequency acoustic volume backscattering in the Georges Bank coastal region and its interpretation using scattering models," IEEE J. Ocean. Eng. 22, 445-463 (1997).

${ }^{38}$ J. A. Taylor, C. E. Vincent, P. D. Thorne, P. J. Hardcastle, V. F. Humphrey, J. Zhang, A. S. Schaafsma, C. M. Dohmen-Janssen, and M. Perennes, "Three-dimensional sediment transport measurements by acoustics (TRIDISMA)," Ocean. Conf. Rec. 2, 1108-1114 (1998).

${ }^{39}$ J. W. Gartner, "Estimating suspended solids concentrations from backscatter intensity measured by acoustic Doppler current profiler in San Francisco Bay, California,” Mar. Geol. 211, 169-187 (2004).

${ }^{40}$ L. I. Costa, G. Storti, B. Lüscher, P. Gruber, and T. Staubli, "Influence of solid particle parameters on the sound speed and attenuation of pulses in ADM," J. Hydrol. Eng. 17, 1084-1092 (2012).

${ }^{41}$ P. D. Thorne, L. Hayhurst, and V. F. Humphrey, "Scattering by nonmetallic spheres," Ultrasonics 30, 15-20 (1992).

${ }^{42}$ L. D. Hampton and C. M. McKinney, "Experimental study of the scattering of acoustic energy from solid metal spheres in water," J. Acoust. Soc. Am. 33, 664-673 (1961).

${ }^{43}$ P. D. Thorne, "Analysis of acoustic measurements of suspended sediments," J. Geophys. Res. 98, 899-910, https://doi.org/10.1029/ 92JC01855 (1993).

${ }^{44}$ D. Hurther, P. D. Thorne, M. Bricault, U. Lemmin, and J. M. Barnoud, “A multi-frequency acoustic concentration and velocity profiler (ACVP) for boundary layer measurements of fine-scale flow and sediment transport processes," Coast. Eng. 58, 594-605 (2011).

${ }^{45}$ P. D. Thorne, S. Sun, J. Zhang, I. Bjorno, and T. Mazoyer, "Measurements and analysis of acoustic backscattering by elastic cubes and irregular polyhedra," J. Acoust. Soc. Am. 102, 2705-2713 (1997).

${ }^{46}$ P. A. Chinnery, V. F. Humphrey, and J. Zhang, "Low-frequency acoustic scattering by a cube: Experimental measurements and theoretical predictions," J. Acoust. Soc. Am. 101, 2571-2582 (1997).

${ }^{47}$ P. A. Chinnery, J. Zhang, and V. F. Humphrey, "Acoustic scattering by nonmetallic and metallic cubes in the elastic resonance regime: Experimental measurements and combined finite element/boundary element modeling," J. Acoust. Soc. Am. 102, 60-66 (1997).
${ }^{48}$ A. E. Hay, "Sound scattering from a particle-laden, turbulent jet," J. Acoust. Soc. Am. 90, 2055-2074 (1991).

${ }^{49}$ A. E. Hay and J. Sheng, "Vertical profiles of suspended sand concentration and size from multifrequency acoustic backscatter," J. Geophys. Res. 97, 15661-15677, https://doi.org/10.1029/92JC01240 (1992).

${ }^{50}$ A. M. Crawford and A. E. Hay, "Determining suspended sand size and concentration from multifrequency acoustic backscatter," J. Acoust. Soc. Am. 94, 3312-3324 (1993).

${ }^{51}$ P. Schurtenberger and A. E. Hay, "Attenuation in suspensions of irregularly shaped sediment particles: A two-parameter equivalent spherical scatterer model,” J. Acoust. Soc. Am. 102, 1485-1502 (1997).

${ }^{52} \mathrm{G}$. W. Wilson and A. E. Hay, "Acoustic backscatter inversion for suspended sediment concentration and size: A new approach using statistical inverse theory," Cont. Shelf Res. 106, 130-139 (2015).

${ }^{53}$ J. Sheng and A. E. Hay, "An examination of the spherical scatterer approximation in aqueous suspensions of sand," J. Acoust. Soc. Am. 83, 598-610 (1988).

${ }^{54}$ T. H. Lee and D. M. Hanes, "Direct inversion method to measure the concentration profile of suspended particles using backscattered sound," J. Geophys. Res. 100, 2649-2657, https://doi.org/10.1029/94JC03068 (1995).

${ }^{55}$ S. D. Richards, A. D. Heathershaw, and P. D. Thorne, "The effect of suspended particulate matter on sound attenuation in seawater," J. Acoust. Soc. Am. 100, 1447-1450 (1996).

${ }^{56}$ G. P. Holdaway and P. D. Thorne, "Determination of a fast and stable algorithm to evaluate suspended sediment parameters from high resolution acoustic backscatter systems," in Proceedings of the Seventh International Conference on Electronic Engineering in Oceanography, Vol. 7, pp. 86-92 (1997).

${ }^{57}$ B. D. Moate and P. D. Thorne, "Measurements and inversion of acoustic scattering from suspensions having broad size distributions," J. Acoust. Soc. Am. 126, 2905-2917 (2009).

${ }^{58}$ B. D. Moate and P. D. Thorne, "Interpreting acoustic backscatter from suspended sediments of different and mixed mineralogical composition," Cont. Shelf Res. 46, 67-82 (2012).

${ }^{59}$ B. D. Moate and P. D. Thorne, "Scattering from suspended sediments having different and mixed mineralogical compositions: Comparison of laboratory measurements and theoretical predictions," J. Acoust. Soc. Am. 133, 1320-1334 (2013).

${ }^{60} \mathrm{D}$. M. Hanes, "On the possibility of single-frequency acoustic measurement of sand and clay concentrations in uniform suspensions," Cont. Shelf Res. 46, 64-66 (2012).

${ }^{61}$ S. A. Moore and A. E. Hay, "Angular scattering of sound from solid particles in turbulent suspension," J. Acoust. Soc. Am. 126, 1046-1056 (2009).

${ }^{62}$ R. Hickling, "Analyis of echoes from a solid elastic sphere in water," J. Acoust. Soc. Am. 34, 1582-1592 (1962).

${ }^{63}$ J. Skripalle, T. Hies, Y. Liu, and H. H. Nguyen, "Application of multifrequency acoustics to estimate concentration of suspended sediments from Jurong Lake, Singapore," in Proceedings of the 17th International Seminar on Hydropower Plants, Institute for Energy Systems and Thermodynamics, Vienna (2012), pp. 725-736.

${ }^{64}$ S. A. Moore, "Monitoring flow and fluxes of suspended sediment in rivers using side-looking acoustic Doppler current profilers," Ph.D. thesis, Université de Grenoble, Grenoble, France (2006).

${ }^{65}$ M. J. W. Povey, "Scattering of sound," in Ultrasonic Techniques for Fluids Characterization (Academic Press, San Diego, 1997), pp. 91-140.

${ }^{66} \mathrm{C}$. E. Vincent, "Measuring suspended sand concentration using acoustic backscatter: A critical look at the errors and uncertainties," Geol. Soc. Spec. Publ. 274, 7-15 (2007).

${ }^{67}$ D. J. McClements, "Principles of ultrasonic droplet size determination in emulsions," Langmuir 12, 3454-3461 (1996).

${ }^{68} \mathrm{D}$. J. McClements and M. J. W. Povey, "Ultrasonic velocity as a probe of emulsions and suspensions," Adv. Colloid Interface Sci. 27, 285-316 (1987).

${ }^{69}$ S. Umchid, "Directivity pattern measurement of ultrasound transducers," Int. J. Appl. Biomed. Eng. 2, 39-43 (1999). 